\title{
Fundamental parameters of Galactic luminous OB stars VI. Temperatures, masses and WLR of Cyg OB2 supergiants ${ }^{\star}$
}

\author{
A. Herrero ${ }^{1,2}$, J. Puls $^{3}$, and F. Najarro ${ }^{4}$ \\ 1 Instituto de Astrofísica de Canarias, 38200 La Laguna, Tenerife, Spain \\ 2 Departamento de Astrofísica, Universidad de La Laguna, Avda. Astrofísico Francisco Sánchez, s/n, 38071 La Laguna, Spain \\ 3 Universitäts-Sternwarte München, Scheinerstr. 1, 81679 München, Germany \\ ${ }^{4}$ Instituto de Estructura de la Materia, Consejo Superior de Investigaciones Científicas, C/ Serrano 121, 28006 Madrid, Spain
}

Received 3 June 2002 / Accepted 30 September 2002

\begin{abstract}
We have analyzed six OB supergiants and one giant covering spectral types from $\mathrm{O} 3$ to $\mathrm{B} 1$ in the Galactic OB association Cyg OB2 by means of an updated version of FASTWIND (Santolaya-Rey et al. 1997) that includes an approximate treatment of metal line blocking and blanketing. This large coverage in spectral type allows us to derive a new temperature scale for Galactic $\mathrm{O}$ supergiants that is lower than the one obtained by using pure $\mathrm{H}-\mathrm{He}$ models, either plane-parallel and hydrostatic or spherical with mass-loss. The lower temperatures are thus a combined effect of line blanketing and the large mass-loss rates. In some cases, the newly derived effective temperature is reduced by up to $8000 \mathrm{~K}$. Changes are larger for earlier stars with large mass-loss rates. As a consequence, luminosities are modified as well, which results in a lower number of emerging ionizing photons and reduces the mass discrepancy. Although there are still significant differences between spectroscopic and evolutionary masses, we do not find any obvious systematic pattern of those differences. We derive mass-loss rates and the corresponding wind momentum-luminosity relation for the analyzed stars. Although consistent with previous results by Puls et al. (1996) for Galactic stars, our relation is better defined due to a reduction of errors related to stellar distances and points to a possible separation between extreme Of stars $\left(\mathrm{Of}^{+}, \mathrm{Of}^{*}\right)$ and stars with more moderate morphologies. However this finding is only tentative, as the statistics are still scarce.
\end{abstract}

Key words. stars: atmospheres - stars: early-types - stars: supergiants - stars: fundamental parameters - Galaxy: open clusters and associations: individual: Cyg OB2 - Ultraviolet: stars

\section{Introduction}

New winds are blowing from hot massive stars. The development of new model atmosphere codes during recent years (Hubeny \& Lanz 1995; Santolaya-Rey et al. 1997; Hillier \& Miller 1998; Pauldrach et al. 2001) employing improved numerical techniques and detailed atomic models offers the opportunity to derive more realistic stellar parameters that can be used for the research of astrophysical problems. Thus very recently Martins et al. (2002) have presented a new temperature scale for massive $\mathrm{O}$ dwarfs that lowers considerably the previous one given by Vacca et al. (1996) as a result of strong metal line blanketing. The effect should be even larger for supergiants, where strong mass-loss should add to metal line opacity to yield even smaller effective temperatures. First indications of this behaviour were obtained for example by Crowther \& Bohannan (1997), Herrero et al. (2000) or Fullerton et al. (2000). More recently, Crowther et al. (2002) have presented

Send offprint requests to: A. Herrero, e-mail: ahd@ll.iac.es

* The INT is operated in the island of La Palma by the ING in the Spanish Observatorio de El Roque de los Muchachos of the Instituto de Astrofísica de Canarias. an analysis of 4 supergiants in the LMC and the SMC with similar trends and Bianchi \& García (2002) obtain comparable results for O6-O7 stars in the Milky Way.

The stellar masses of $\mathrm{O}$ stars are still a subject of debate. A large part of the uncertainty originates from the so-called mass-discrepancy (Herrero et al. 1992): masses derived from a spectroscopic analysis using hydrostatic, plane-parallel model atmospheres are systematically lower than those predicted by non-rotating evolutionary models. Unified model atmospheres are expected to reduce the discrepancy, especially if new evolutionary models account for rotationally induced mixing in stars (Herrero et al. 2000; Meynet \& Maeder 2000). To this end, considerable effort has been made in the last decade to account for the possibility of He enriched and CNO contaminated atmospheres in early type stars. Efforts to improve the derived abundances have lowered them, but without completely ruling out He enhancements (Villamariz et al. 2002).

On the other hand, the presence of a wind momentumluminosity relation (WLR) for hot, massive stars offers the unique opportunity to derive stellar distances directly from the analysis of the observed spectra with an accuracy of about $10 \%$ (Kudritzki et al. 1999). For this purpose, the coefficients 
in the relation

$\log \left(\dot{M} V_{\infty} R^{0.5}\right) \approx \frac{1}{\alpha^{\prime}} \log L+C=x \log \left(L / L_{\odot}\right)+\log D_{0}$

have to be calibrated as function of metallicity and spectral type. For O-stars, this has been done by Puls et al. (1996) for Galactic and MC objects. However, their WLR shows a large scatter, especially for non-supergiants. It is reasonable to assume that part of this scatter results from uncertainties in the individual stellar distances, as their sample was mainly taken from Herrero et al. (1992), aiming at targets with large apparent brightness.

Following this idea, we started a programme to calibrate the WLR using a more homogeneous sample of OB supergiants, all of them belonging to the same OB association. From this approach we expected to reduce the scatter in the derived WLR. The OB association chosen was Cyg OB2, and the optical observations and first analysis using plane-parallel, hydrostatic models were presented in Herrero et al. (1999). The required wind terminal velocities were obtained from HST STIS observations and are discussed in Herrero et al. (2001).

By means of spherical, mass-losing models we will derive here an improved WLR for those Cyg OB2 supergiants that were observed with the HST. (One of the stars, Cyg OB2 \#4, is actually a luminosity class III object, but we will refer to our sample as Cyg OB2 supergiants. When required, we will emphasize the difference.)

This effort is being complemented in our group by other studies to calibrate the WLR in the Local Group (see McCarthy et al. 1997; Urbaneja et al. 2002; Bresolin et al. 2002 for M 31 and M 33).

The remainder of this paper is organized as follows. In Sect. 2 we describe the characteristics of the code we have used, and in Sect. 3 we present an analysis of the O9 V star $10 \mathrm{Lac}$ as a test case. The analyses of our targets is given in Sect. 4, and we end with a discussion of our results in Sect. 5 and the conclusions in Sect. 6.

\section{The code}

The analyses presented here have been performed by means of the latest version of FASTWIND (an acronym for Fast Analysis of STellar atmospheres with WINDs), a code which was originally described by Santolaya-Rey et al. (1997) and was recently updated to include an approximate treatment of metal line opacity effects, i.e., metal line blocking and blanketing.

The code follows the philosophy of using suitable physical approximations allowing for a fast computational time, thus enabling us to calculate a large number of models (which are necessary to analyze stellar atmospheres with winds), while being realistic enough to preserve their value as a tool for determining stellar parameters.

It assumes a $\beta$-velocity law in the wind and calculates a consistent photospheric structure; the temperature structure is approximated using "NLTE Hopf functions" as described in Santolaya-Rey et al. (1997); the coupling between the radiation field and the rate equations is treated within an ALI scheme, using local ALOs following Puls (1991). The program allows for a solution of the rate equations using either Sobolev or comoving frame calculations, but all calculations presented here were done using comoving frame. The formal solution to calculate the emergent profiles utilizes a radial micro-grid to account for the different scales involved and includes Stark broadening, which is a prerequesite for the analysis of $\mathrm{O}$ stars by means of $\mathrm{H}$ and $\mathrm{He}$ lines.

The approximate treatment of metal line blocking/blanketing will be described in detail by Puls (2002), in the following we will give only a brief summary. The basic philosophy to calculate the required NLTE metal opacities (bound-bound, bound-free and free-free) follows the line of reasoning given by Abbott \& Lucy (1985), Schmutz (1991), Schaerer \& Schmutz (1994) and Puls et al. (2000), however applying significant improvements. In particular and most important for realistic results, we have reformulated the equations of approximate ionization equilibrium (e.g., Puls et al. 2000, Eq. (43)) to account for the actual radiation field (as function of depth) at all ionization edges (including those from excited levels) and employ a consistent coupling of rate-equations and mean intensity, in a way similar to the ALI approach, to avoid any kind of Lambda Iteration.

The underlying atomic data base has been described by Pauldrach et al. (1998). In order to save computational effort, the resulting metal line opacities are averaged in a suitable way (mean of inverse opacities, in analogy to Rosseland means) over a frequency interval of order wind terminal velocity before the radiation transport is performed. Finally, flux conservation (and thus line blanketing) is obtained by adapting the NLTEHopf parameters in a consistent way. The method has been carefully tested by comparing the results with up-to-date methods, in particular with calculations performed with TLUSTY (Hubeny \& Lanz 1995) for the case of dwarfs (see also the next section) and with WMBasic (Pauldrach et al. 2001) for the case of stars with winds.

\section{A test case: 10 Lac}

Before applying the modified code to the analysis of our Cyg OB2 sample, we have analyzed the O9V star 10 Lac as a test case. This star is well suited for calibration purposes because it is a luminosity class $\mathrm{V}$ star with a low projected rotational velocity, has already been used as standard by Herrero et al. (1992) and was later considered by Hubeny et al. (1998) to study the effects of line blanketing in plane-parallel, hydrostatic atmospheres.

Figure 1 gives the fit diagram for the $\mathrm{H} / \mathrm{He}$ spectrum of 10 Lac. It is very similar to the corresponding fit diagram in Herrero et al. (1992), but now it is centered at a lower effective temperature (and a slightly lower gravity). A comparison of both diagrams also reveals that the dispersion around the final model is now smaller. Therefore, the present error box has a width of only $1000 \mathrm{~K}$ instead of $2000 \mathrm{~K}$, as the one in Herrero et al. (1992). It is reassuring that all lines, including He II $\lambda 4200$ that could not be fitted by Herrero et al. (1992), lie within the error box.

The stellar parameters of $10 \mathrm{Lac}$ we have derived here are $T_{\text {eff }}=35500 \pm 500 \mathrm{~K}, \log g=3.95 \pm 0.10$ 


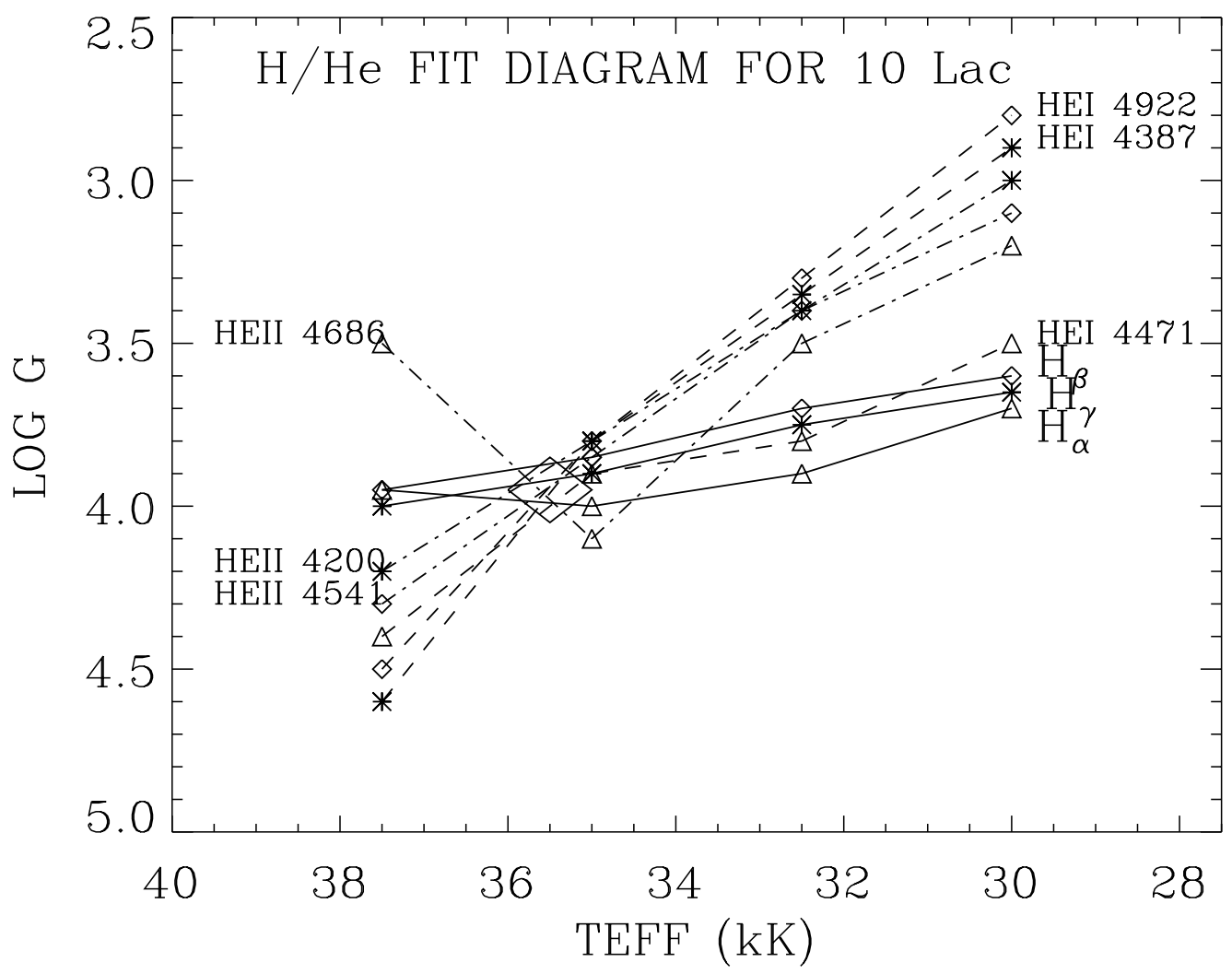

Fig. 1. Fit diagram to the $\mathrm{H} / \mathrm{He}$ spectrum of $10 \mathrm{Lac}$ using FASTWIND plus approximated line blocking/blanketing. The filled circle marks the location of the chosen model, and the box around gives the size of the adopted error box.

and $\epsilon=\frac{N(\mathrm{He})}{N(\mathrm{H})+N(\mathrm{He})}=0.09 \pm 0.03, N(\mathrm{X})$ being the abundance of element $\mathrm{X}$ by number. The only significant difference compared to the results from Herrero et al. (1992) is the effective temperature, now lower by $2000 \mathrm{~K}$. This is in complete agreement with the result obtained by Hubeny et al. (1998). These authors estimated a temperature of $35000 \mathrm{~K}$ for $10 \mathrm{Lac}$ using TLUSTY, a plane-parallel, hydrostatic, line-blanketed model.

Fit diagrams have the drawback that they only give the best possible fit for the chosen constraints. They rely on interpolations and sometimes (when using $E W \mathrm{~s}$ ) do not account for the profile shape. The actual final fit may still be poor. Figure 2 shows the line spectra for our final model of 10 Lac. Good agreement is found for all lines, although a few details are not perfectly reproduced. In particular, the core of He II $\lambda 4200$ is too weak, which is also true for the forbidden component of Не г $\lambda 4471$. Besides this, however, the final fit is perfectly consistent in all other aspects.

Our result also agrees with the study of Martins et al. (2002), who recently found that using pure $\mathrm{H} / \mathrm{He}$ models (as Herrero et al. 1992 did) results in an effective temperature scale for O dwarfs hotter by 1500 to $4000 \mathrm{~K}$ compared to using line-blanketed models.

These authors have derived a new effective temperature scale for O dwarfs using CMFGEN (Hillier \& Miller 1998), a spherical code including mass-loss and blanketing. In their $T_{\text {eff }}$ scale, O9V stars are located at $33000 \mathrm{~K}$. However, their scale is calibrated using the equivalent width $(E W)$ - spectral classification relations of Conti \& Alschuler (1971) and
Mathys (1998), and thus we have to compare our result with Martins et al. using the classification of 10 Lac in this system.

Conti \& Alschuler (1971) have classified 10 Lac as O8 III and not as a luminosity class V star (which it has been considered by Martins et al. 2002), although the star lies just at the border between both luminosity classes. The luminosity class III is mainly due to the low $E W$ quoted by Conti \& Alschuler (1971) for the He I $\lambda 4143$ line. Other EWs quoted by these authors are consistent with our observations which show a much larger value for this line, resulting in a luminosity class $\mathrm{V}$ within their classification scheme. Thus, we conclude that 10 Lac should be classified as $\mathrm{O} 8 \mathrm{~V}$ in the system of Conti \& Alschuler (1971) (whatever the reason for the low $E W$ in Conti \& Alschuler 1971 was).

The effective temperature in the Martins et al. scale for $\mathrm{O} 8$ dwarfs lies between 36000 and $35000 \mathrm{~K}$, in perfect agreement with our result. Therefore we regard our result as fully consistent with recent findings using more elaborate but also more expensive fully blanketed NLTE model atmospheres.

Nevertheless we have analyzed 10 Lac with our version of CMFGEN that includes a consistent photospheric structure (Najarro 2002) and have fitted the line profiles instead of only using their $E W \mathrm{~s}$. Our results from CMFGEN completely agree with those from FASTWIND.

The reason for the lower temperatures derived is twofold (see also Martins et al. 2002). On the one hand, the radiation field which is backscattered due to the additional opacity produces a larger $(E) \mathrm{UV}$ radiation field. On the other hand, due to 

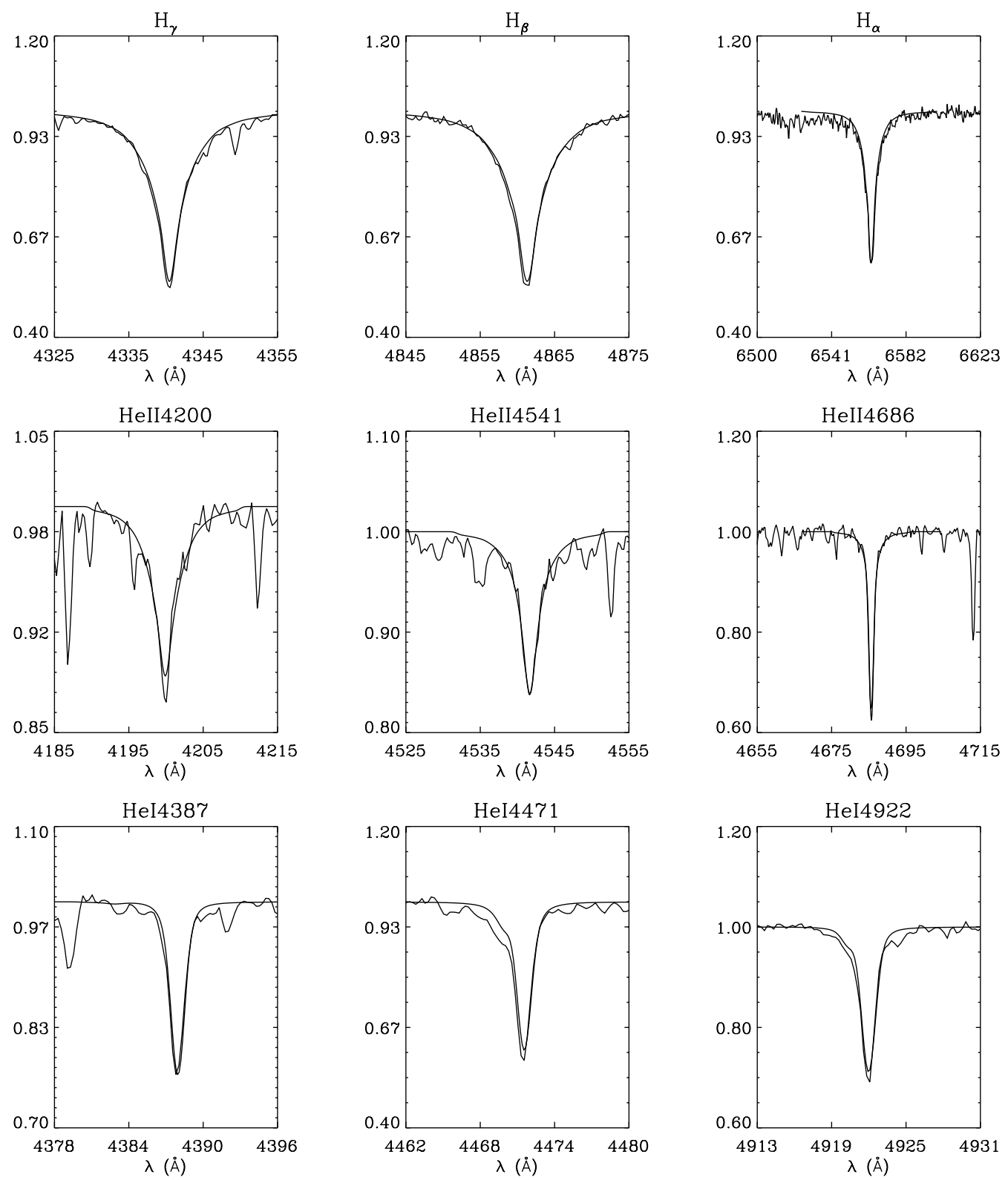

Fig. 2. The fit to the H/He spectrum of 10 Lac using FASTWIND with approximated line blocking/blanketing. The ordinate gives the relative flux values. Note that the scales are different for each line. See text for comments.

line-blanketing the electron temperature rises in photospheric regions. Both effects favour a higher ionization degree at lower effective temperatures, compared to unblanketed models. This effect can be clearly seen in Fig. 3 .

The analysis of 10 Lac gives us an indication of what we can expect when introducing metal line opacity (namely lower effective temperatures), either in the form of traditional lineblanketing (as Hubeny et al. 1998; Martins et al. 2002 and references therein) or including the line-blanketing via adapted Hopf-parameters (as here). Although with our method we do not force flux conservations, in all models calculated here the flux is conserved to better than $3 \%$ at all depths, where the remaining deviations have no impact on the emergent fluxes and profiles.

Our analysis of $10 \mathrm{Lac}$ also gives us an idea of the error bar we can expect for the stellar parameters. For a resolution of 8000 , a $S / N$ of 200 and a projected rotational velocity of $40 \mathrm{~km} \mathrm{~s}^{-1}$, the estimated errors are $\pm 500 \mathrm{~K}$ in $T_{\text {eff }}, \pm 0.1 \mathrm{dex}$ in $\log g$ and \pm 0.03 in $\epsilon$. For what follows we shall note here that this analysis does not give us information about the mass loss rate or the $\beta$ exponent in the wind velocity law, as the wind of $10 \mathrm{Lac}$ is negligible. We obtain an upper limit of $10^{-8} M_{\odot} \mathrm{yr}^{-1}$, but the fit has the same quality for any value below that limit. (The fit presented here was performed with $\dot{M}=10^{-10} M_{\odot} \mathrm{yr}^{-1}$.)

\section{Analysis of the Cyg OB2 supergiants}

We make use of optical and UV spectra that have been presented elsewhere (Herrero et al. 1999; Herrero et al. 2001). The optical spectra have been newly rectified, resulting in a less pronounced bump in the 4630-4700 $\AA$ region, which, however, does not affect our present analysis. Basic data, together with previously determined parameters adopted here are listed in 


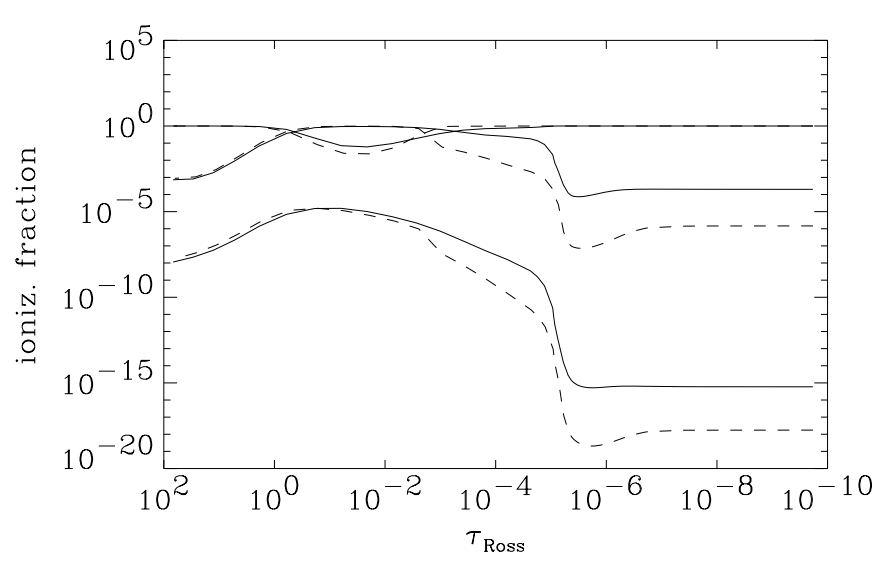

Fig. 3. The He ionization fractions in two models with $T_{\text {eff }}=$ $35500 \mathrm{~K}, \log g=3.95$ and $\epsilon=0.09$, with approximated lineblocking/blanketing (solid line) and without (dashed line). At typical photospheric line formation depths the ionization degree of the model including metal opacities is larger. Thus, lower temperatures are required in these models to reproduce the He spectrum.

Table 1, while the new parameters determined in this work are provided in Table 2. Note that the gravities in that table have been corrected for the effect of centrifugal acceleration (in the same way as in Herrero et al. 1992). This effect is small (modifying only the last digit of the entries in our table) and the gravities actually used in our calculations are always given by the nearest lower value ending with " 0 " or " 5 " in the second decimal (i.e., a corrected value of 3.52 corresponds to a model value of 3.50). The corrected gravities have been used for the calculation of stellar masses.

Errors adopted for the parameters given in Table 2 have been taken from the errors obtained for Cyg OB2 \#7 (see below) or from those for $10 \mathrm{Lac}$, depending on the stellar parameters, the spectrum quality and the fit conditions. A summary of all errors is given in Table 3.

For Cyg OB2 \#7, errors have been estimated "by eye" from a microgrid of models around the final one. At fixed $\beta$ (the exponent of the velocity law), this resulted in $\pm 1000 \mathrm{~K}$ in $T_{\text {eff }}$, ${ }_{-0.10}^{+0.15}$ dex in $\log g, \pm 0.05$ dex in $\epsilon$ and ${ }_{-0.10}^{+0.05}$ dex in $\dot{M} . \beta$ is determined from the form of the $\mathrm{H}_{\alpha}$ wings, and its uncertainty is estimated to be \pm 0.10 . This has an influence on the derived effective temperature and mass-loss rates. Therefore, the adopted error for $T_{\text {eff }}$ has been increased to $\pm 1500 \mathrm{~K}$ and that for $\dot{M}$ to ${ }_{-0.15}^{+0.10}$.

The errors for radii and masses depend on the error in the absolute magnitude. This is assumed to be \pm 0.1 from the work by Massey \& Thompson (1991). (The influence of the error in $T_{\text {eff }}$ on the stellar radius is marginal and has been neglected here.) For the analysis we have adopted a microturbulence of $10 \mathrm{~km} \mathrm{~s}^{-1}$. Tests indicate that this parameter is of no relevance for the results presented here.

In the following we will comment on the individual analyses. Further discussions about the individual stars were presented in Herrero et al. (2001).

Cyg OB2 \#7 The final fit to the observed $\mathrm{H} / \mathrm{He}$ spectrum of Cyg OB2 \#7 is shown in Fig. 4. The good fit to the $\mathrm{H}_{\alpha}$ profile is accompanied by a much poorer fit to the other two Balmer lines (and also to $\mathrm{H}_{\delta}$, not displayed here). This behaviour reproduces the one found in Herrero et al. (2000): for stars with strong winds we could not obtain a consistent fit for all Balmer lines at a given mass-loss rate. The situation has improved with the new version of our code, but the discrepancy still reaches a $30 \%$ effect, by which the mass-loss rate has to be reduced (from $10^{-5}$ to $7.7 \times 10^{-6}$ ) in order to fit $\mathrm{H}_{\beta}$ and $\mathrm{H}_{\gamma}$. The other stellar parameters are not affected by this modification, as the fit to the other lines does not change. We adopt the massloss rate indicated by $\mathrm{H}_{\alpha}$ as this line is much more sensitive to changes in this parameter and there is good general agreement between the mass-loss rates from $\mathrm{H}_{\alpha}$ and radio fluxes (Scuderi et al. 1998). Our result also supports this conclusion, as the mass-loss rate derived here agrees with the upper limit of $1.5 \times 10^{-5} M_{\odot} \mathrm{yr}^{-1}$ quoted by Bieging et al. (1989) $\left(1.6 \times 10^{-5}\right.$ if we use our values for distance and $v_{\infty}$ ) in case the star is a thermal emitter (the authors classify the object among the probable thermal emitters). However, in addition to the error quoted, $\dot{M}$ could be lowered by an additional $20 \%$ if we would adopt the value indicated by $\mathrm{H}_{\gamma}$.

The singlet He I lines $(\lambda \lambda 4387,4922)$ give a poor fit to the observed spectrum, partly due to the difficulties in the continuum rectification. Therefore we have given a low weight to these lines when determining the stellar parameters. However, since these lines react strongly to changes in stellar parameter, it is always possible to find a reasonable fit within the error box. The error in $\epsilon$ is larger than for other stars and $\epsilon$ itself is not well constrained towards higher He abundances, because the already large He abundance produces a saturation effect.

The derived $T_{\text {eff }}$ is much lower than the one obtained by Herrero et al. (2000) using the same code as here but without line-blanketing. The derived luminosity is also lower by more than 0.2 dex, as the radius does not change very much. The reason can be seen in Fig. 5, where we compare the emergent energy of two models for Cyg OB2 \#7. The first model (the dash-dotted line in the figure) is the one adopted here, with a $T_{\text {eff }}$ of $45500 \mathrm{~K}$, a radius of $14.6 R_{\odot}$ and metal line opacity. The second model (solid line) corresponds to the model adopted by Herrero et al. (2001), with a $T_{\text {eff }}$ of $50000 \mathrm{~K}$, a radius of $14.8 R_{\odot}$ and pure $\mathrm{H} / \mathrm{He}$ opacities. Both models give the same optical luminosity and thus fit equally well the observed visual magnitude of Cyg OB2 \#7. We additionally show in the figure the CMFGEN luminosities calculated with the same parameters and conditions (dashed lines). The good agreement supports our approximated treatment of the metal line opacity.

The reason for the similarity in derived radii is that the emergent flux is strongly blocked in the UV by the metal line opacity and thus emerges at higher wavelengths, including the optical. Therefore, at lower temperatures we obtain larger optical fluxes for models that include metal line opacity than for pure $\mathrm{H} / \mathrm{He}$ models of the same temperature.

The radius needed to fit the observed visual magnitude is then significantly smaller for models with metal line opacities (compared to unblanketed models at the same $T_{\text {eff }}$ ), however roughly similar to the "old" value derived from unblanketed models at higher $T_{\text {eff }}$. In consequence, the reduction in luminosity is mostly due to the change in the effective temperature. 
Table 1. Cyg OB2 supergiants studied in this work. All numerical identifications are taken from Schulte (1958). Magnitudes have been adopted from Massey \& Thompson (1991), as well as spectral types, except for Cyg OB2 \#11 and \#4, that are taken from Walborn (1973). $S / N$ values have been estimated from the rms of the continuum at several wavelength intervals. Velocities are given in $\mathrm{km} \mathrm{s}^{-1}$.

\begin{tabular}{rrccccc}
\hline \hline Ident & $\begin{array}{r}V \\
\text { mag. }\end{array}$ & $\begin{array}{c}\text { Spectral } \\
\text { Type }\end{array}$ & $M_{v}$ & $S / N$ & $V_{\mathrm{r}} \sin i$ & $v_{\infty}$ \\
& 10.55 & O3 If $^{\star}$ & -5.91 & 140 & 105 & 3080 \\
11 & 10.03 & O5 If $^{+}$ & -6.51 & 190 & 120 & 2300 \\
$8 \mathrm{C}$ & 10.19 & O5 If & -5.61 & 195 & 145 & 2650 \\
$8 \mathrm{~A}$ & 9.06 & O5.5 I(f) & -7.09 & 135 & 95 & 2650 \\
4 & 10.23 & O7 III((f)) & -5.44 & 230 & 125 & 2550 \\
10 & 9.88 & O9.5 I & -6.86 & 145 & 85 & 1650 \\
2 & 10.64 & B1 I & -4.64 & 195 & 50 & 1250 \\
\hline
\end{tabular}

Table 2. Results obtained using FASTWIND plus line blocking/blanketing. Effective temperatures have been derived from the He ionization equilibrium. Gravities include the correction for centrifugal acceleration. $\epsilon$ is the He abundance by number relative to $\mathrm{H}$ plus $\mathrm{He} ; R, L$ and $M$ are given in solar units. $M_{\mathrm{s}}, M_{\mathrm{ev}}$ and $M_{0}$ are the spectroscopic, present evolutionary and initial evolutionary masses, the two latter from the models by Schaller et al. (1992). $\dot{M}$ is given in solar masses per year, and $\log M W M$ means the logarithm of the Modified Wind Momentum rate, $\dot{M} v_{\infty} R^{0.5}$

\begin{tabular}{rllllllllllll}
\hline \hline Ident & $\begin{array}{l}\text { Spectral } \\
\text { Type }\end{array}$ & $T_{\text {eff }}$ & $\log g$ & $\epsilon$ & $R$ & $\beta$ & $\dot{M}$ & $\log L$ & $M_{\mathrm{s}}$ & $M_{\mathrm{ev}}$ & $M_{0}$ & $\log M W M$ \\
\hline 7 & O3 If & 45.5 & 3.71 & 0.23 & 14.6 & 0.90 & $9.86 \mathrm{e}-6$ & 5.91 & 39.7 & 67.4 & 69. & 29.864 \\
11 & O5 If $^{+}$ & 37.0 & 3.61 & 0.09 & 22.2 & 0.90 & $9.88 \mathrm{e}-6$ & 5.92 & 73.0 & 58.1 & 63. & 29.829 \\
$8 \mathrm{C}$ & O5 If & 41.0 & 3.81 & 0.08 & 13.3 & 0.90 & $2.25 \mathrm{e}-6$ & 5.66 & 42.2 & 46.1 & 48. & 29.137 \\
$8 \mathrm{~A}$ & O5.5 I(f) & 38.5 & 3.51 & 0.09 & 27.9 & 0.70 & $1.35 \mathrm{e}-5$ & 6.19 & 90.5 & 78.4 & 95. & 30.076 \\
4 & O7 III((f)) & 35.5 & 3.52 & 0.09 & 13.6 & 1.00 & $8.60 \mathrm{e}-7$ & 5.41 & 21.8 & 32.6 & 34. & 28.708 \\
10 & O9.5 I & 29.0 & 3.11 & 0.09 & 30.3 & 1.00 & $3.09 \mathrm{e}-6$ & 5.77 & 43.1 & 43.7 & 48. & 29.248 \\
2 & B1 I & 28.0 & 3.21 & 0.09 & 11.3 & 1.00 & $6.92 \mathrm{e}-8$ & 4.85 & 7.5 & 18.1 & 19. & 27.263 \\
\hline
\end{tabular}

Table 3. Errors adopted for the parameters given in Table 2, in the same units as in that table. If only one value is provided, it should be preceeded by the \pm sign. See text for comments.

\begin{tabular}{|c|c|c|c|c|c|c|c|c|c|c|c|c|}
\hline \multirow[t]{2}{*}{ Ident } & Spectral & $\Delta$ & $\Delta$ & $\Delta$ & $\Delta$ & $\Delta$ & $\Delta$ & $\Delta$ & $\Delta$ & $\Delta$ & $\Delta$ & $\Delta$ \\
\hline & Type & $T_{\text {eff }}$ & $\log g$ & $\epsilon$ & $\log R$ & $\beta$ & $\log \dot{M}$ & $\log L$ & $M_{\mathrm{s}}$ & $M_{\mathrm{ev}}$ & $M_{0}$ & $\log M W M$ \\
\hline 7 & O3 If* & 1.5 & $\begin{array}{l}+0.15 \\
-0.10\end{array}$ & $\begin{array}{l}+0.10 \\
-0.05\end{array}$ & 0.02 & 0.10 & $\begin{array}{l}+0.10 \\
-0.15\end{array}$ & 0.10 & $\begin{array}{l}+17 \\
-13\end{array}$ & $\begin{array}{l}+11 \\
-8\end{array}$ & ${ }_{-8}^{+11}$ & $\begin{array}{l}+0.12 \\
{ }_{-0.17}\end{array}$ \\
\hline 11 & O5 $\mathrm{If}^{+}$ & 1.5 & $\begin{array}{l}+0.15 \\
-0.10\end{array}$ & 0.03 & 0.02 & 0.10 & $\begin{array}{l}+0.10 \\
-0.15\end{array}$ & 0.11 & $\begin{array}{l}+32 \\
-24\end{array}$ & $\begin{array}{l}+10 \\
-9\end{array}$ & $\begin{array}{l}+11 \\
-10\end{array}$ & $\begin{array}{l}+0.12 \\
-0.17\end{array}$ \\
\hline $8 \mathrm{C}$ & O5 If & 1.5 & 0.10 & 0.03 & 0.02 & 0.10 & $\begin{array}{l}+0.10 \\
-0.15\end{array}$ & 0.10 & 14 & 5 & 5 & $\begin{array}{l}+0.12 \\
-0.17\end{array}$ \\
\hline $8 \mathrm{~A}$ & O5.5 I(f) & 1.5 & 0.10 & 0.03 & 0.02 & 0.10 & $\begin{array}{l}+0.10 \\
-0.15\end{array}$ & 0.11 & 29 & $\begin{array}{l}+4 \\
-7\end{array}$ & 15 & $\begin{array}{l}+0.12 \\
-0.17\end{array}$ \\
\hline 4 & O7 III((f)) & 1.0 & 0.10 & 0.03 & 0.02 & 0.10 & 0.10 & 0.09 & 7 & 4 & 5 & 0.12 \\
\hline 10 & O9.5 I & 1.0 & 0.10 & 0.03 & 0.02 & 0.10 & 0.10 & 0.10 & 14 & $\begin{array}{l}+12 \\
-6\end{array}$ & $\begin{array}{l}+16 \\
-6\end{array}$ & 0.13 \\
\hline 2 & B1 I & 1.0 & 0.10 & 0.03 & 0.04 & 0.10 & 0.10 & 0.14 & 3 & 3 & 3 & 0.13 \\
\hline
\end{tabular}

Note, however, the dramatic difference of the ionizing fluxes in the $(E) \mathrm{UV}$.

The helium abundance derived here is even larger than the one obtained by Herrero et al. (2000), although the error bars overlap significantly. Cyg OB2 \#7 is thus confirmed as the only star in our sample for which we derive an enhanced He abundance. The $\beta$ value we obtain (0.9) is slightly larger than the one obtained from the UV analysis presented in Herrero et al. (2001), a behaviour which has been found already in previous investigations (e.g., Puls et al. 1996). However, our mass-loss rate is similar to the one derived in that work, resulting again from the fact that the optical fluxes are similar.

Finally, the $\log g$ value obtained here is slightly larger than the one obtained by Herrero et al. (2000). This results in a spectroscopic mass of $39.7 M_{\odot}$, to be compared with an evolutionary mass of $67.4 M_{\odot}$. Accounting for maximum errors, masses of 56.7 and $59.4 M_{\odot}$, respectively, are possible, still not overlapping. However, we have to remember the large $\mathrm{He}$ abundance derived for Cyg OB2 \#7. This is an indication that this star might be evolved or be affected by rotational mixing. 

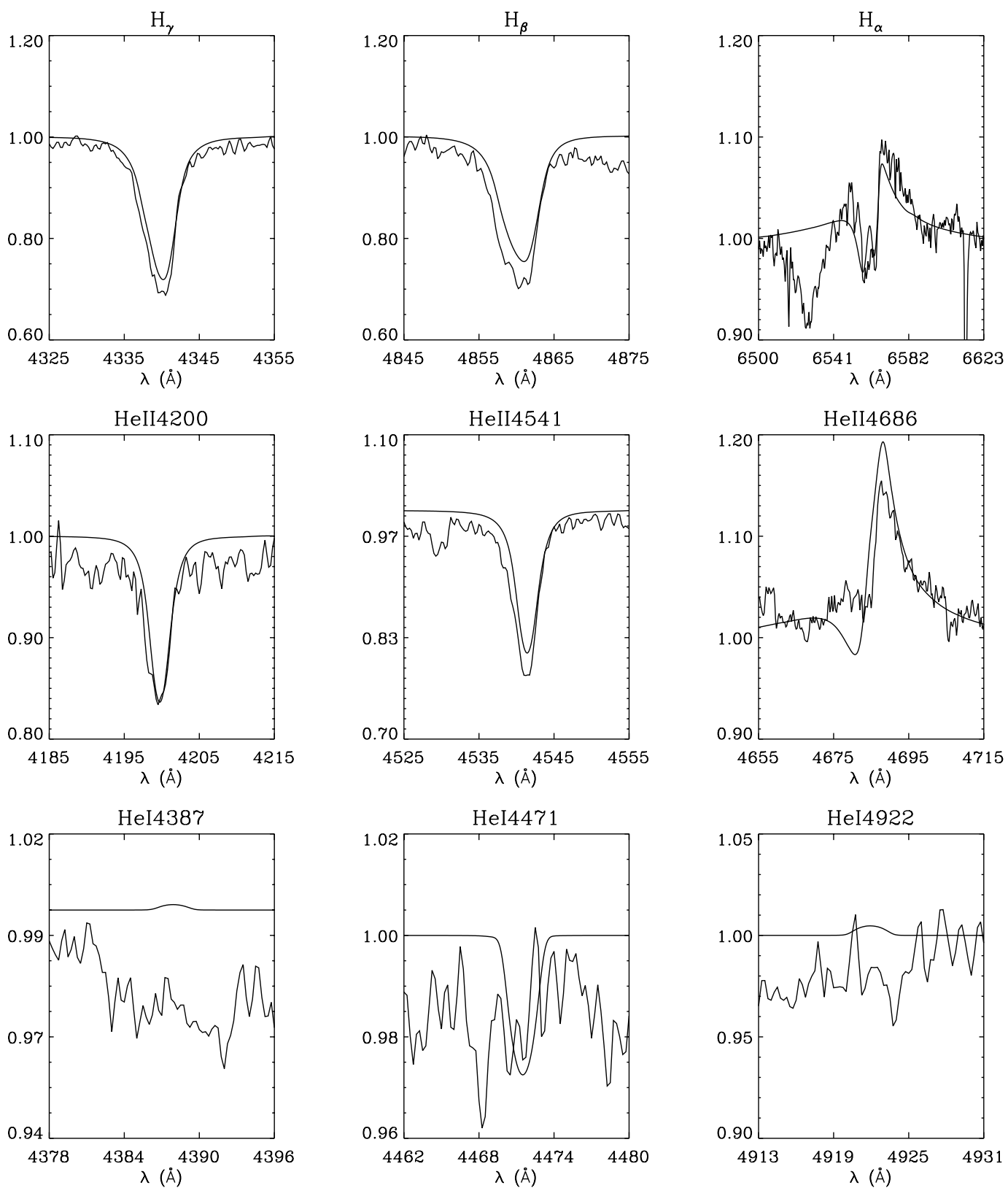

Fig. 4. As Fig. 2, however for CygOB2 \#7. See text for comments.

Taken together, there is no (clear) evidence that evolutionary and spectroscopic masses really disagree.

Walborn et al. (2000) and Walborn et al. (2002) have recently studied Cyg OB2 \#7 and HD 93 129A ${ }^{1}$. They conclude, from a comparison of their spectra, that Cyg OB2 \#7 has to be cooler than HD 93 129A, and in fact HD 93 129A has been reclassified as the prototype of the new O2 If* class. Taresch et al. (1997) have analyzed this latter star and obtained an effective temperature of $52000 \pm 1000 \mathrm{~K}$, based on the $\mathrm{N} \mathrm{v} / \mathrm{N} \mathrm{IV} / \mathrm{N}$ III ionization equilibrium. This is similar to the Herrero et al. (2000) value and again much higher than the temperature we obtain here for Cyg OB2 \#7. Both stars display simultaneously Nv, N IV and $\mathrm{N}_{\text {III }}$ lines in their spectra, and thus we would not expect a very large temperature difference. Clearly, a

\footnotetext{
1 Note added in proofs: Walborn (2002, Proc. IAU Symp. 212) reports that HD $93129 \mathrm{~A}$ is a binary.
}

cross-calibration of $\mathrm{He}$ and $\mathrm{N}$ blanketed temperature scales for the earliest stars is an urgent task.

Cyg OB2 \#11 The fit to Cyg OB2 \#11 is given in Fig. 6. It shows the same problems as the fit to Cyg OB2 \#7, except for the fit to the He I singlet lines, which are again affected by continuum rectification problems. However their depths relative to the depressed local continuum are now well predicted.

Therefore we adopt the same errors, except for $\epsilon$, for which we adopt \pm 0.03 . It is interesting that in spite of the extreme Of character of both stars and the similarity of the problems found, we do not derive an enhanced He abundance for Cyg OB2 \#11. This star also shows the same trend as Cyg OB2 \#7 towards cooler temperatures and lower luminosities, but now the spectroscopic and evolutionary masses (73.0 and 58.1 $M_{\odot}$, respectively) invert their usual ratio. When considering the formal 


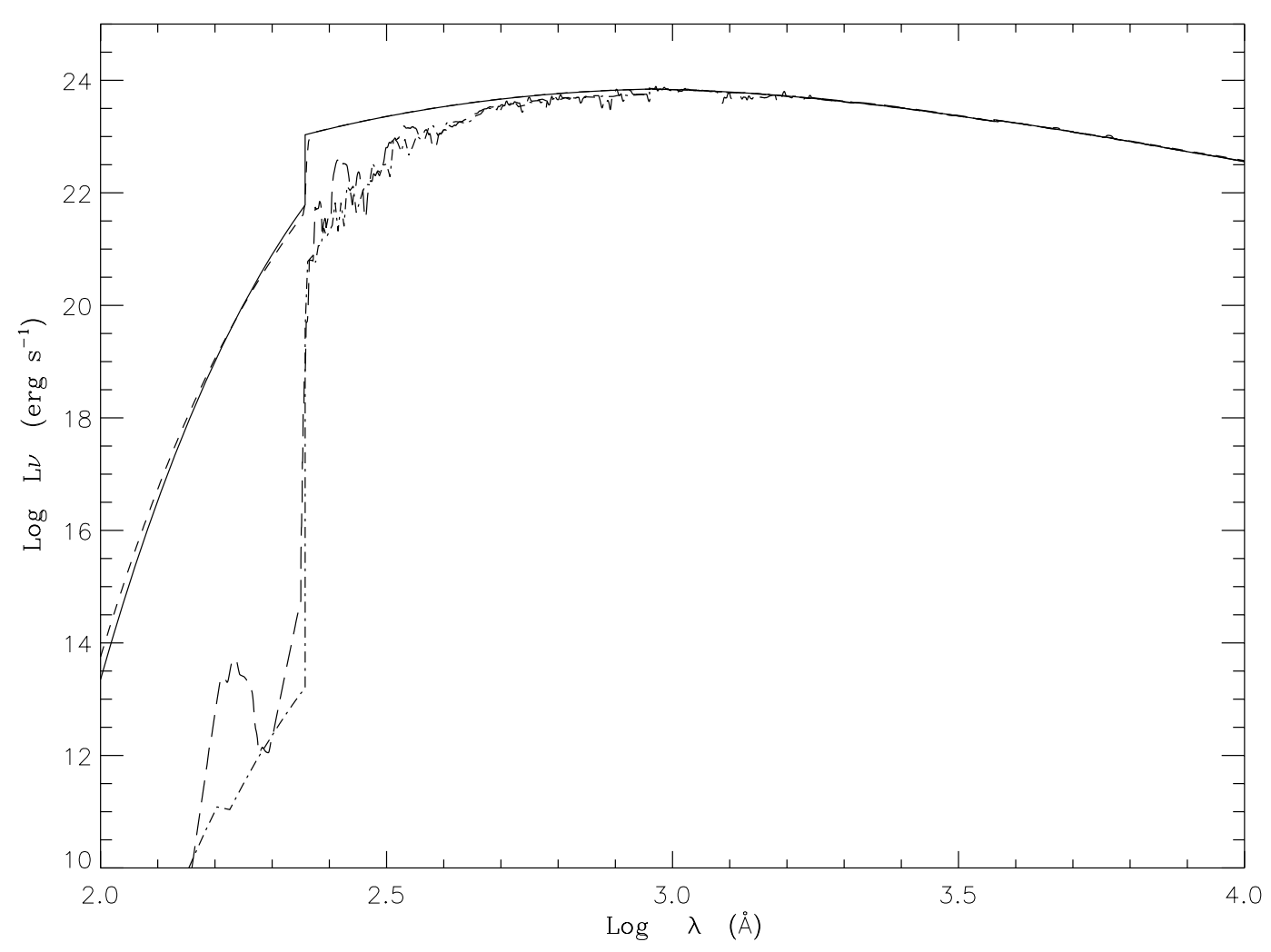

Fig. 5. Emergent energy of two models for Cyg OB2 \#7, each one calculated both with FASTWIND and CMFGEN. The first pair of models corresponds to the one adopted here including metal line opacity and is represented by the lower dashed (CMFGEN) and dash-dotted (FASTWIND) lines. The second pair corresponds to the model adopted by Herrero et al. (2001) that did not include metal line opacity and is represented by the upper dashed (CMFGEN) and solid (FASTWIND) lines. We see that all models give the same optical flux, but very different UV fluxes (see text). We also see the good agreement between FASTWIND and CMFGEN, although individual strong UV resonance lines are not visible in the former due to the approximate method applied.

errors presented in Table 3, the mass ranges of both stars overlap significantly.

The $\beta$ value we have used is again 0.9 . The mass loss rate we derive is consistent with the upper limit given by Bieging et al. (1989) $\left(1.4 \times 10^{-5} M_{\odot} \mathrm{yr}^{-1}\right.$, or $1.2 \times 10^{-5}$ using again our values for distance and wind terminal velocity).

Cyg OB2 \#8C The fit to Cyg OB2 \#8C is presented in Fig. 7. The only problem is a serious failure in the predicted He II $\lambda 4686$ line (which is not used in the fit procedure). To fit this line one had to increase the mass-loss rate by at least a factor of 1.7, although we note that the observed line lies at top of a broad emission feature that we cannot reproduce. The same comments as for Cyg OB2 \#11 apply for the He I singlet lines.

$\beta$ is not well constrained from the wings of $\mathrm{H}_{\alpha}$, as these are in absorption. We have adopted the same value as for Cyg OB2 \#7 and \#11 (0.9), as well as the same errors.

The resulting mass-loss rate is consistent with the upper limit quoted by Bieging et al. (1989) of $8.8 \times 10^{-6} M_{\odot} \mathrm{yr}^{-1}$. The gravity is large for a supergiant (a lower gravity is prohibited by the Balmer line wings), but we find good agreement between the spectroscopic and evolutionary masses.

Cyg OB2 \#8A The final fit can be seen in Fig. 8. The fit to He II $\lambda 4686$ is problematic, but much less than for Cyg OB2 \#8C, while the same comments apply for the He I singlet lines. Errors are the same as for Cyg OB2 \#8C. The temperature is cooler and the luminosity lower than quoted in Herrero et al. (2001). However, the spectroscopic mass is again very large $\left(90.5 M_{\odot}\right)$, larger than the evolutionary one (78.4 $M_{\odot}$ ), but with significant overlap when considering the errors.

The mass-loss rate we derive here is nearly a factor of two lower than the one given in Herrero et al. (2001). Note, that the latter was not derived from spectrum analysis, however was calculated from the luminosity (believed to be larger at that time) and the Galactic WLR.

Our new mass-loss rate in Table $2\left(1.35_{-0.39}^{+0.35} \times\right.$ $\left.10^{-5} M_{\odot} \mathrm{yr}^{-1}\right)$ agrees well with the radio mass-loss rate given by Waldron et al. (1998) $\left(1.97 \times 10^{-5}\right)$, and lies between the extreme values one would derive from the fluxes given by Bieging et al. (1989) $\left(1.1-6.1 \times 10^{-5}\right)$ assuming free-free emission. Although Cyg OB2 \#8A is a known non-thermal emitter, with variable radio flux and spectral index (Waldron et al. 1998; Bieging et al. 1989), our $\mathrm{H}_{\alpha}$ mass-loss rate is of the same order of magnitude as the radio mass-loss rates and consistent with their lower limit. This consistency contradicts the suggestion by Waldron et al. (1998) that the X-ray emission might originate from an X-ray source deeply embedded in the stellar wind, i.e., a base corona model scenario, which would imply a much lower mass-loss rate $\left(\approx 1.5 \times 10^{-6} M_{\odot} \mathrm{yr}^{-1}\right)$. 

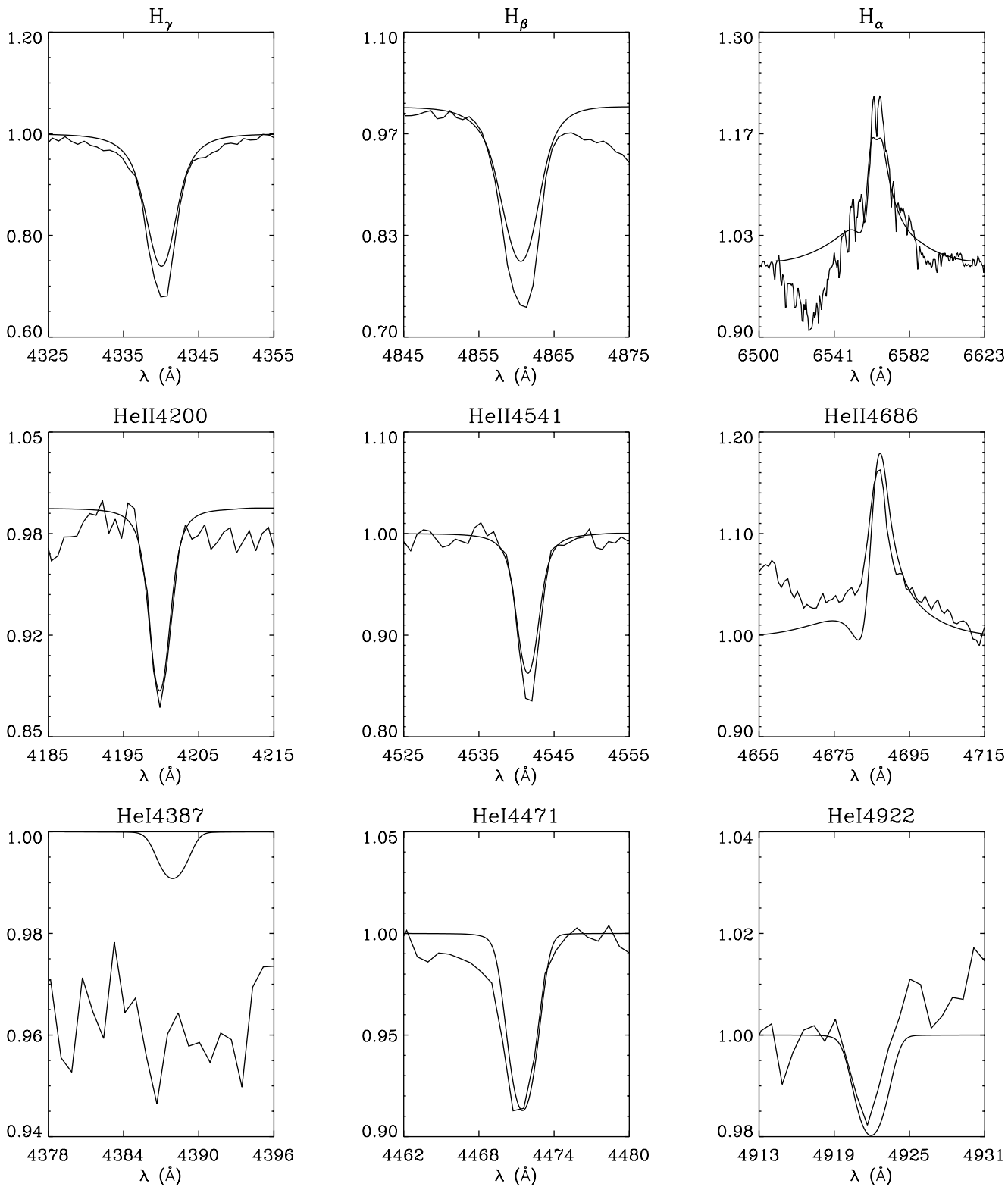

Fig. 6. As Fig. 2, however for CygOB2 \#11. See text for comments.

Cyg OB2 \#4 The fit to Cyg OB2 \#4 is presented in Fig. 9. The predicted He II $\lambda 4686$ and the singlet He I line at $\lambda 4922$ are too strong in the core, although we note the large scale in the corresponding plots. The fit of He r $\lambda 4387$ is acceptable taking the normalization into account. $\beta$ is again not well constrained from the $\mathrm{H}_{\alpha}$ wings and we adopt a similar value as for the cooler stars in our sample $(\beta=1)$. However, the influence of $\beta$ on the other stellar parameters begins to decrease and therefore we adopt the same errors as for $10 \mathrm{Lac}$.

The mass loss rate is not well constrained towards lower values, because the profiles react only slightly. In this case, as also for the next two Cyg OB2 stars, there are no radio mass-loss rates available to compare with (which is an indication of a rather low value). The derived effective temperature is still cooler than in Herrero et al. (2001), although the differences begin to decrease. The evolutionary and spectroscopic mass ranges agree within the large error bars.
Cyg OB2 \#10 The fit to Cyg OB2 \#10 is given in Fig. 10. As for Cyg OB2 \#4, the main difficulties appear in the fit of the $\mathrm{He}$ II $\lambda 4686$ and the He I lines, where from the three He I lines one is slightly too strong, the second slightly too weak and the third one fits well. The errors adopted are the same as for Cyg OB2 \#4. The derived effective temperature is still lower than in Herrero et al. (2001), but the difference is only $2000 \mathrm{~K}$. The spectroscopic and evolutionary masses agree well.

Cyg OB2 \#2 The final fit to the star is shown in Fig. 11. The adopted errors are the same as for Cyg OB2 \#8C (because of the lower $S / N$ compared to $10 \mathrm{Lac}$ ), although again the mass loss rate is not well constrained towards lower values. Here, the derived effective temperature is hotter than in Herrero et al. (2001), but also the derived He abundance has decreased significantly. The masses, however, do not agree, with 

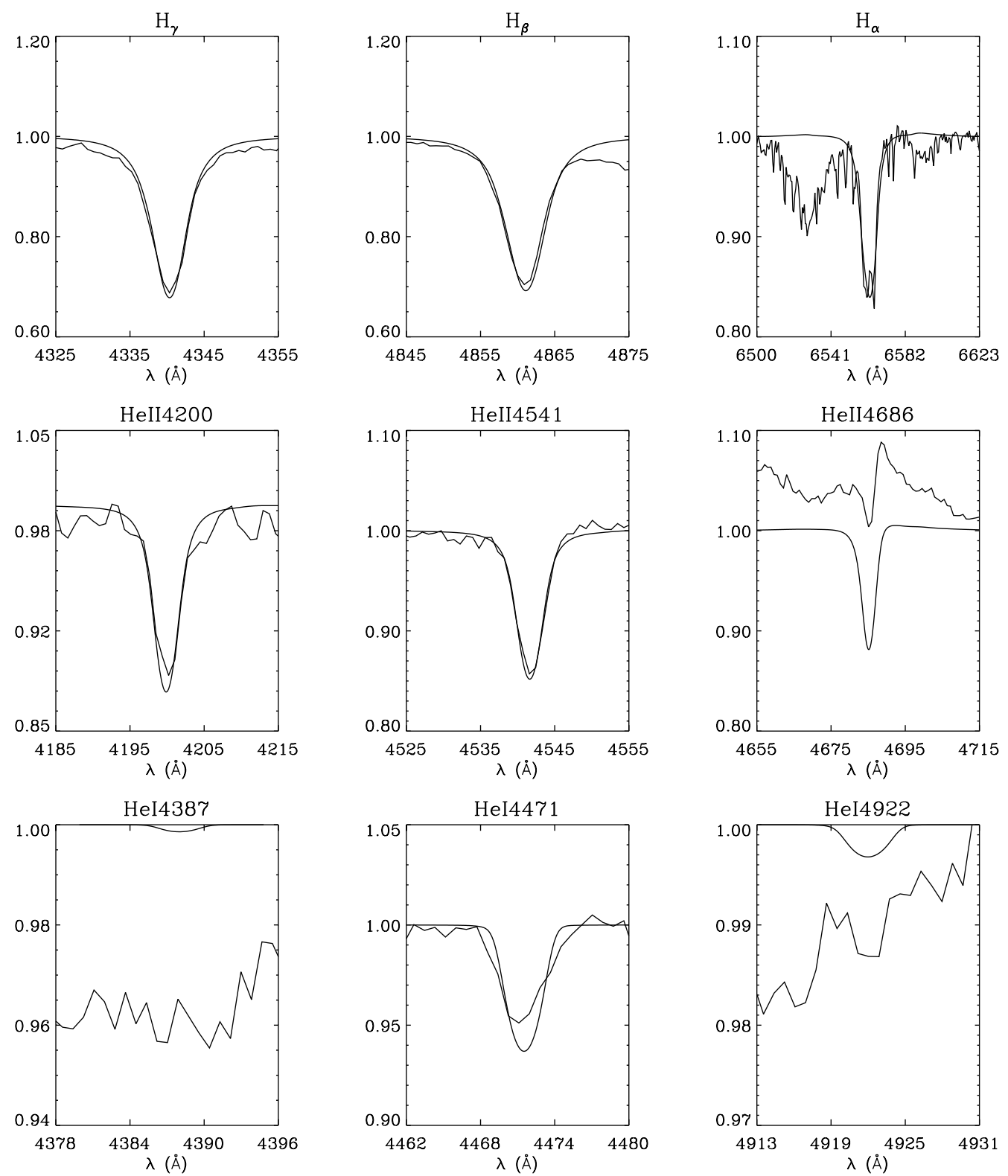

Fig. 7. As Fig. 2, however for CygOB2 \#8C. See text for comments.

the spectroscopic mass being lower than the evolutionary one by a factor of two.

Herrero et al. (2001) indicated some problems with the stellar classification as B1 I and its Cyg OB2 membership, because this would result in a rather faint absolute magnitude for its spectral class. However, the large reddening quoted by Massey \& Thompson (1991) indicates that the star is probably related to or lies beyond Cyg OB2. On the other hand, this large reddening is comparatively low when compared to other Cyg OB2 members, which additionally weakens the above argument. (Cyg OB2 \#2 has the fourth lowest reddening in Table 7 of Massey \& Thompson 1991, who list a total 64 Cyg OB2 stars. The stars with the three lowest values lie in the same region of the association as Cyg OB2 \#2). Thus, we adopt the absolute magnitude derived from the canonical distance to Cyg OB2 and assume a larger error, \pm 0.2 instead of \pm 0.1 , which also doubles the error in the (logarithmic) radius.

\section{Discussion}

The results obtained in the preceding section allow us to address four important aspects of the properties of massive OB supergiants: the effective temperature scale, the ionizing fluxes, the mass discrepancy and the wind momentumluminosity relationship.

\subsection{The effective temperature scale for massive $O$ supergiants}

With the inclusion of mass-loss, sphericity and lineblocking/blanketing we derived lower temperatures than those quoted in Herrero et al. (2001), where we have used planeparallel, hydrostatic models without line-blocking for all stars except Cyg OB2 \#7, for which a spherical model with massloss, but without line-blocking was used. As we have covered 

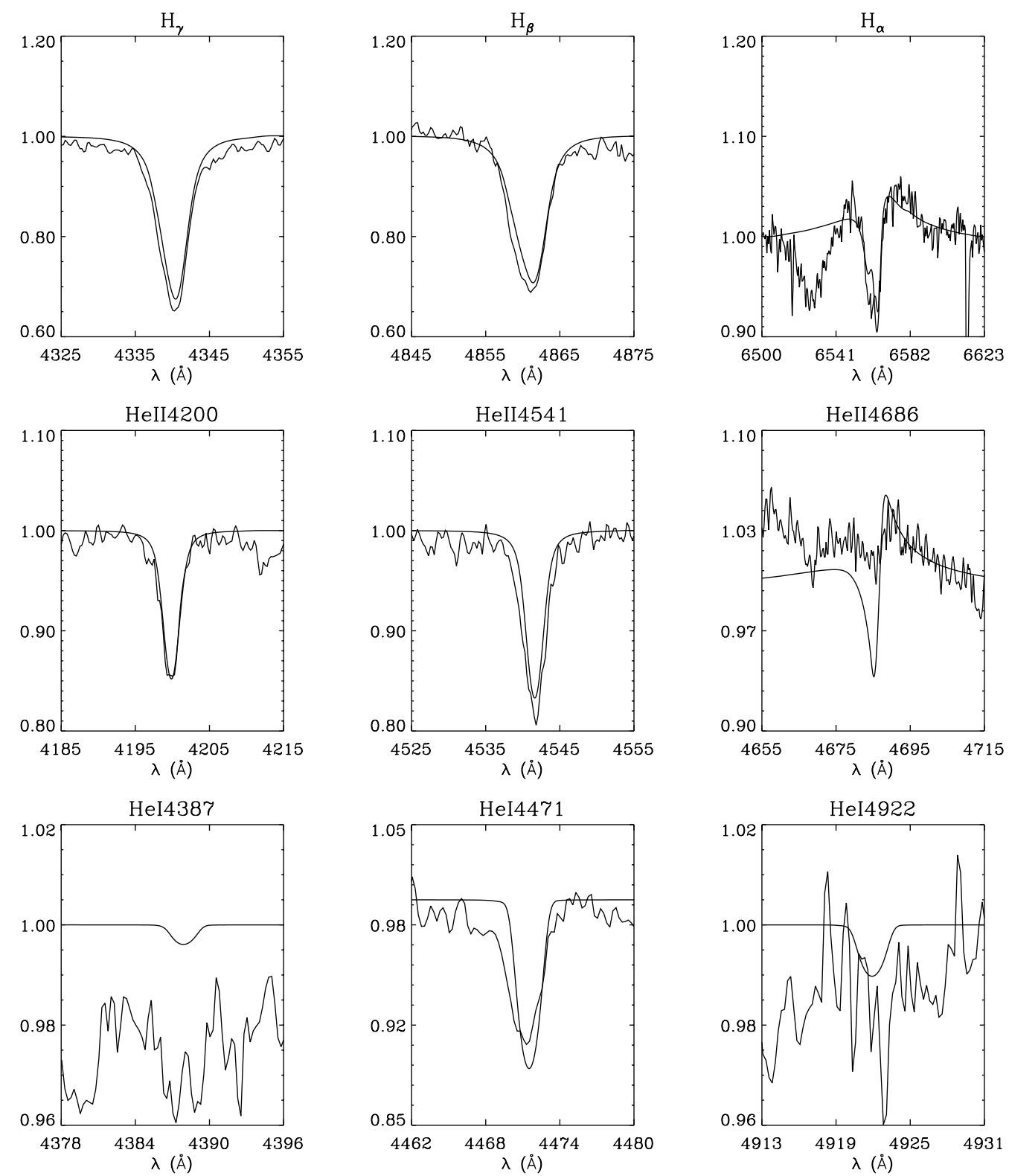

Fig. 8. As Fig. 2, however for CygOB2 \#8A. See text for comments.

various spectral types from $\mathrm{O} 3$ to $\mathrm{B} 1$ in our analysis, we can obtain a temperature scale for $\mathrm{O}$ supergiants. We note however, that Cyg OB2 \#4 is actually a luminosity class III star, while \#2 is probably of class II.

For our earlier supergiants (O3-O7) we find temperatures that are 4000 to $8000 \mathrm{~K}$ cooler than in Herrero et al. (2001), while for the O9.5 I supergiant we obtain $2000 \mathrm{~K}$ less and for the B1 star the temperature is now hotter, but with a lower $\mathrm{He}$ abundance. These findings are in qualitative agreement with theoretical expectations (e.g., Schaerer \& Schmutz 1994) and with the recent temperature scale for $\mathrm{O}$ dwarfs presented by Martins et al. (2002). These authors obtain lower temperatures for O dwarfs by $1500-4000 \mathrm{~K}$ when including line-blanketing and sphericity.

Our temperature scale is shown in Fig. 12, together with the one from Vacca et al. (1996) for O supergiants. The effective temperature scale from these authors has been mainly derived from analyses using pure $\mathrm{H}-\mathrm{He}$, plane-parallel, hydrostatic models, like those performed by Herrero et al. (1992). It is thus not surprising that our new scale is cooler.

In spite of the still low number statistics, we already appreciate some interesting features in Fig. 12. First, from O3 to O9 we see a smooth temperature decline into which Cyg OB2 \#4 fits perfectly, despite its luminosity class III. From this smoothness is excluded Cyg OB2 \#11, much cooler than the other O5 supergiant, Cyg OB2 \#8C, and even cooler than the O5.5 supergiant, Cyg OB2 \#8A. The main difference in their properties is the extreme Of character of Cyg OB2 \#11, which thus appears to be related to cooler temperatures. This is indicating to us that all spectral signatures have a significance in terms of stellar parameters, and thus a temperature scale using only spectral subtypes of $\mathrm{O}$ supergiants, with the various nuances in their classification scheme, will neccesarily be of limited accuracy. 

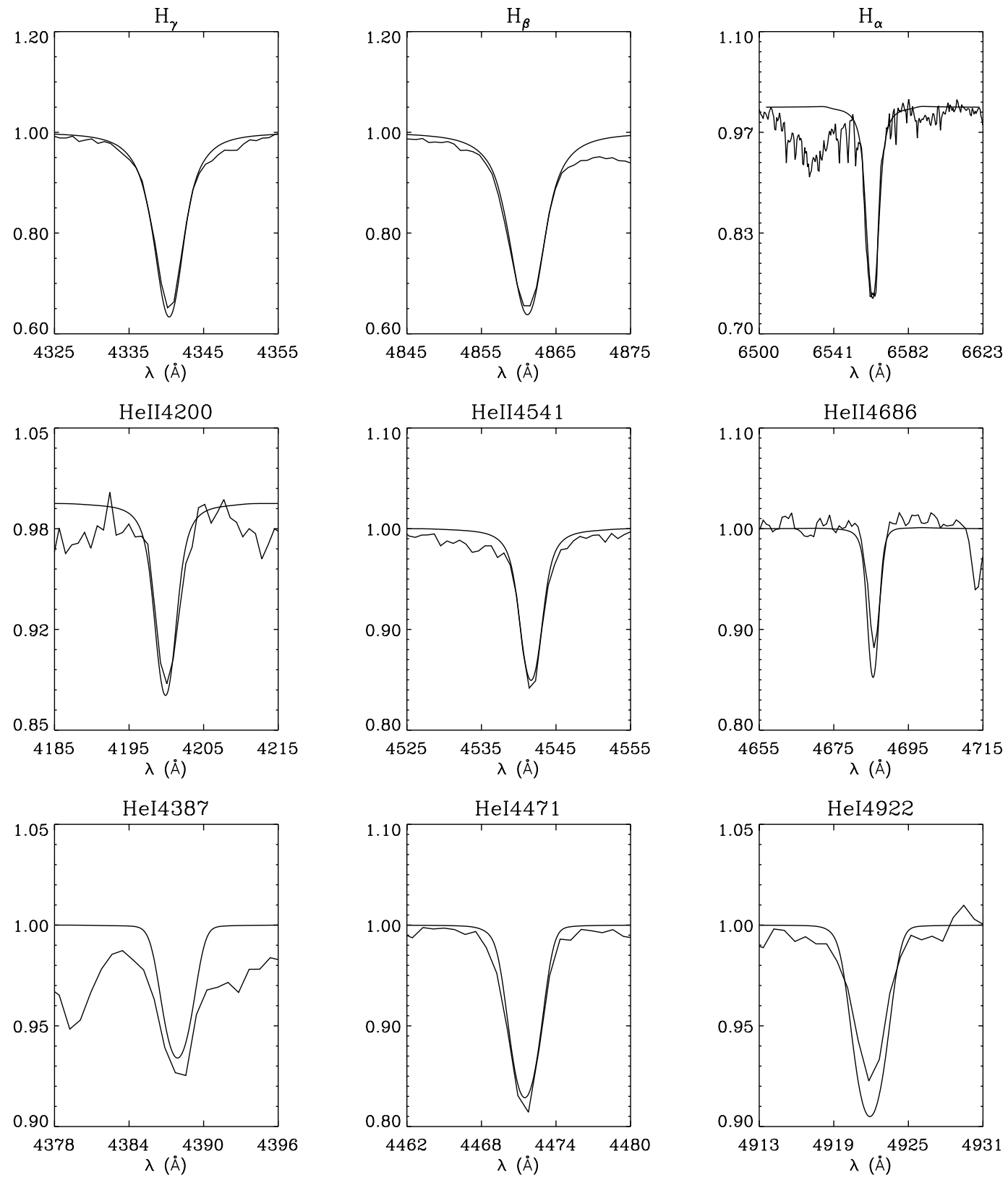

Fig. 9. As Fig. 2, however for CygOB2 \#4. See text for comments.

We also see that both temperature scales converge towards later spectral types, until the B1 star, Cyg OB2 \#2. This object has a temperature that does not seem to fit into the general behaviour, although data are still too scarce to know whether this has any significance.

The new temperature scale and the lower luminosities will have an influence on other aspects, e.g., on the emergent ionizing fluxes that are now much lower than in the older models, as we have seen in the discussion of Cyg OB2 \#7.

\section{2. lonizing fluxes from $O$ supergiants}

Our treatment of UV metal line opacity is approximate (in the sense that we use an approximate NLTE approach and suitably averaged lines opacities), and we do not pretend to give a detailed description of the UV radiation field. However, the emergent fluxes, should be correct in an average sense (i.e., neglecting distinct spectral features, see again Fig. 5), in particular concerning frequency integrated quantities. (Note that the differences bluewards from HeII $228 \AA$ are mostly due to different temperature structures in the outer wind.) Thus, we should obtain a rather correct description of quantities extending over broad spectral regions, like the number of photons capable of ionizing hydrogen.

This number, of course, is of extreme relevance to studies of H II regions surrounding the stars. Vacca et al. (1992) have calculated ionizing fluxes from plane-parallel, hydrostatic, LTE, line-blanketed Kurucz-models (Kurucz 1992) and concluded, from a comparison with more elaborate models available at that time (Schaerer \& Schmutz 1994), that 

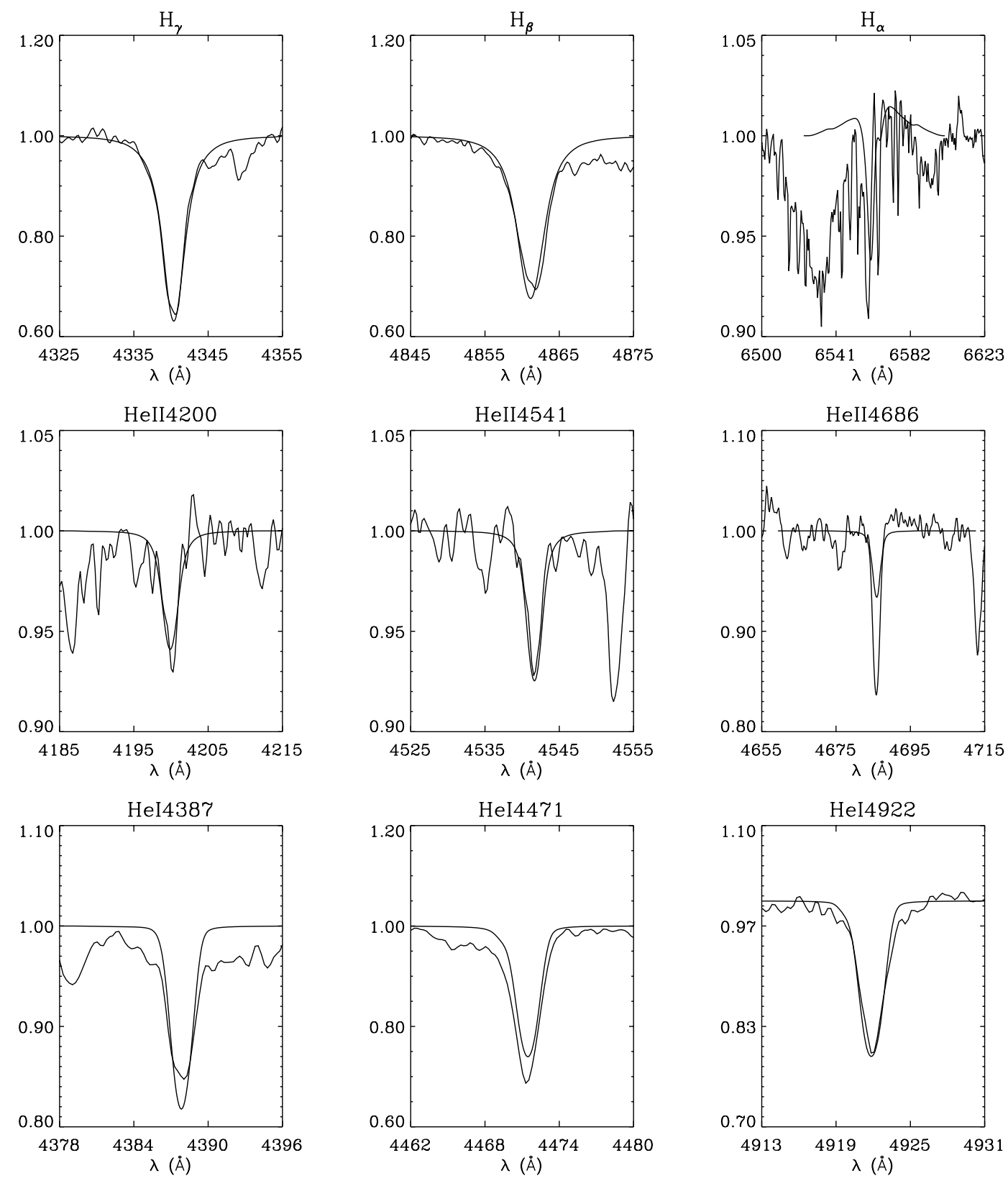

Fig. 10. As Fig. 2, however for CygOB2 \#10. See text for comments.

their ionizing fluxes should be reliable. That would indicate that line-blocking/blanketing is the major effect when calculating the UV continuum ionizing flux at a given effective temperature.

Our calculations seem to support this conclusion, but also indicate that for an application to $\mathrm{H}_{\text {II }}$ regions further effects have to be considered.

In Fig. 13a we have plotted our calculated $\mathrm{H}$ ionizing fluxes versus the effective temperature of the corresponding model. The dashed line gives the calibration by Vacca et al. (1996) for $\mathrm{O}$ supergiants (their Table 7). We see that the agreement is good. Even more, the entry departing slightly from the relation corresponds to Cyg OB2 \#4, that has a luminosity class III. Note that the ionizing fluxes are basically independent of stellar radius. Thus, at given temperature the effects of metal line opacity remain the major ingredient which detemine the emergent UV flux, and the differences between NLTE and LTE models seem to be small regarding the integrated hydrogen Lyman fluxes. (This behaviour should become significantly different when, considering, e.g., He II ionizing fluxes because of the extreme sensitivity of this ion to NLTE effects, in particular as function of mass-loss.)

In Fig. 13b we have plotted the $\mathrm{H}$ ionizing luminosities as function of spectral type. Here, the differences with the calibration by Vacca et al. (1996) are apparent. They clearly originate from our new relation between spectral type and effective temperature (and our lower radii for most stars, compared to the calibrations by Vacca et al. 1996), i.e., the stellar luminosities at given subtype are now smaller. (This discrepancy between both plots, of course, results from the former inconsistency of 

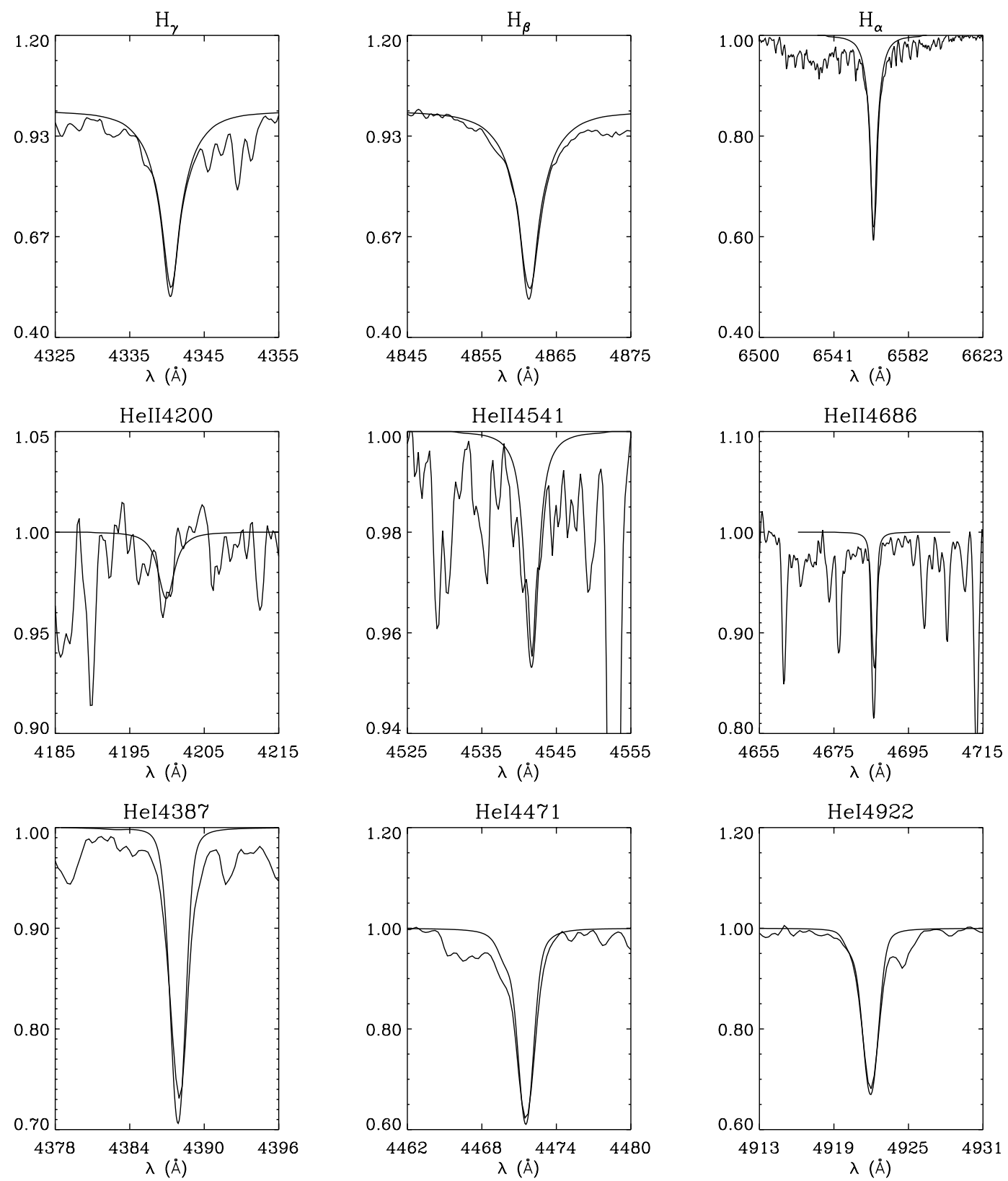

Fig. 11. As Fig. 2, however for CygOB2 \#2. See text for comments.

calibrating spectral types via unblanketed models, however calculating the number of ionizing photons from blanketed ones). Furthermore, for one case (Cyg OB2 \#8A) we find that our ionizing luminosities are larger as a consequence of the larger radius in this particular case. Since the ionizing luminosity is the quantity which really matters for the ionization of $\mathrm{H}$ II regions, our results indicate that statistically there are fewer photons available compared to earlier findings, but also that individual cases have to be studied in detail, because they can depart from the general trend.

\subsection{The mass discrepancy}

The mass discrepancy is a term that was used by Herrero et al. (1992) to refer to the systematic difference between spectroscopically determined stellar masses of OB supergiants and their evolutionary counterparts. The latter were always systematically larger, well beyond the error bars.

In Fig. 14 we have plotted the evolutionary mass (derived from the "classical" models by Schaller et al. (1992) without rotation) and the spectroscopic masses obtained in our analysis for the Cyg OB2 supergiants. We see that the situation is by no means satisfactory: despite the (very) large errors adopted for the spectroscopic masses, three of the seven stars still do not cross the one-to-one relation and for two other we find only a marginal agreement. In fact, only two out of the seven stars have masses that agree reasonably well. However, compared to previous diagrams the situation seems to have improved: there is no clear systematic trend any longer. Roughly the same number of data points lie on each side of the one-to-one relation and the apparent scatter might be related to problems in the individual analyses. 


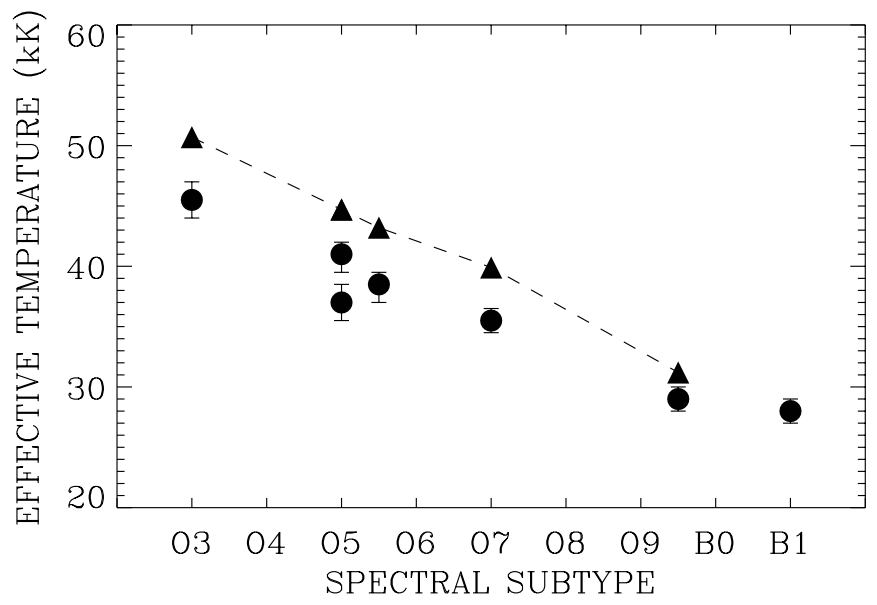

Fig. 12. Our temperature scale for O supergiants (circles) compared with the scale by Vacca et al. (1996) (triangles). New temperatures are much lower, except for the relatively cool Cyg OB2 \#2 (B1I). Note that the entry at $\mathrm{O} 7$ is actually a luminosity class III star.
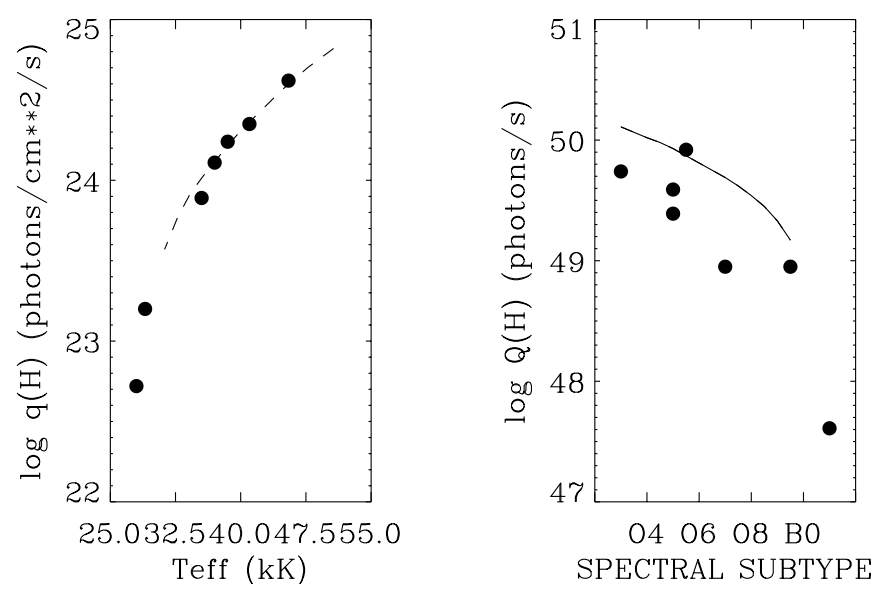

Fig. 13. a) left: ionizing fluxes (photons $\mathrm{cm}^{-2} \mathrm{~s}^{-1}$ ) at the stellar surface versus effective temperature. b) right: ionizing luminosities (photons $\mathrm{s}^{-1}$ ) versus spectral type. Dots correspond to the stellar analyses presented here and lines represent the calibrations by Vacca et al. (1996) for O supergiants.

Nevertheless it is somewhat too early to conclude that the atmosphere models with sphericity, mass-loss and metal opacity agree with the evolutionary models without rotation, and thus give the same answer in a statistical sense. First, the large scatter in Fig. 14 still poses a question for the masses of both set of models; and second, the stars in Cyg OB2 have moderate projected rotational velocities and are very young, with only the earliest type exhibiting an enhanced He abundance. Thus, older or faster rotating stars may have masses that disagree even more when derived using different methods.

This result indicates that we badly need both a calibration of present mass scales based on early type binaries (or any other reliable method), and CNO abundances for isolated massive stars.

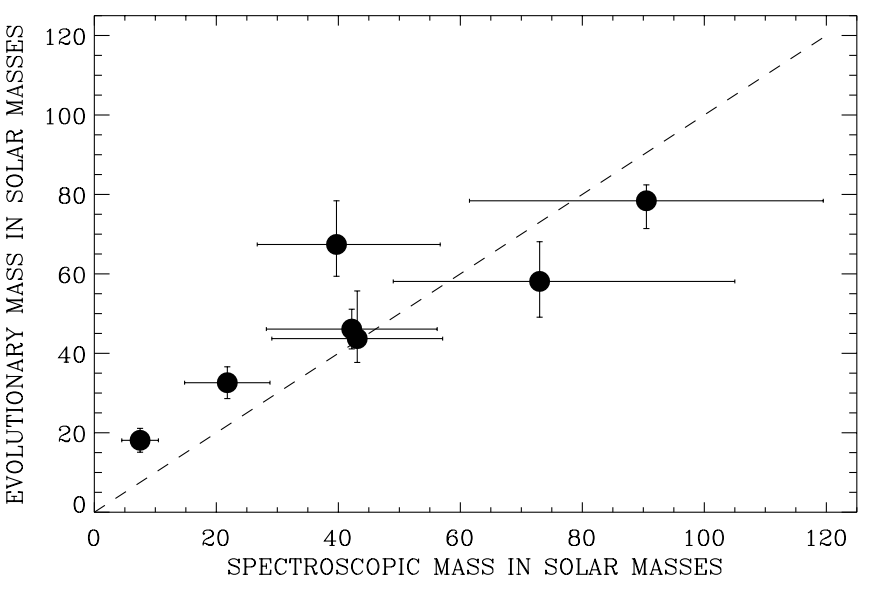

Fig. 14. A comparison of the spectroscopic and evolutionary masses for the Cyg OB2 supergiants. Although there are still serious differences, no obvious systematic trend is present. See text for details and discussion.

\subsection{The Wind momentum-Luminosity Relationship in Cyg OB2}

The original purpose of our work was to obtain a better constrained WLR for Galactic O stars by using objects belonging to the same association, and thus minimizing the scatter introduced by uncertainties in the relative distances. The distance to Cyg OB2 and thus the derived luminosities may be in error, but all determinations will be affected in a similar way.

Figure 15 displays the WLR obtained for our Cyg OB2 sample. Errors for $v_{\infty}$ are taken from Herrero et al. (1991). The stars \#8A, \#8C, \#4 and \#10 follow a nice sequence with low scatter. Cyg OB2 \#7 and \#11 seem to lie above this sequence. This is interesting because these stars display the most extreme Of signatures in their spectra, which might be related to an ionization change in the wind that could result in a different line-driving force or to clumping effects that would produce an overestimation of the mass-loss rate. Cyg OB2 \#2 seems to lie below that relation, which is consistent with the results by Kudritzki et al. (1991), who found a different WLR (with a lower offset) for the winds of early B supergiants, compared to the O-star case. Our observed relation also agrees well with the theoretical WLR derived by Vink et al. (2000) (the dashed line in Fig. 15).

We have investigated whether a reduction in the outer minimum electron temperature (that the model is allowed to reach, see Santolaya-Rey et al. 1997, Sect. 3.1) might result in lower mass-loss rates for Cyg OB2 \#7 and \#11. While we usually assume that $T_{\min }=0.75 T_{\text {eff }}$ (a typical value for OB stars), calculations by Pauldrach (private communication) indicate that this value can reach values as low as $0.40 T_{\text {eff }}$ in extreme cases. This effect has usually no influence on an analyis as performed here, where almost all considered lines are formed in a region with temperatures beyond this minimum. "Only" $\mathrm{H}_{\alpha}$ (and He II 24686) might be affected in cases of extreme massloss, which is the reason that we have investigated here this question. Nevertheless, in all considered cases the resulting reduction of the derived mass-loss rate was less than $20 \%$. Thus, 


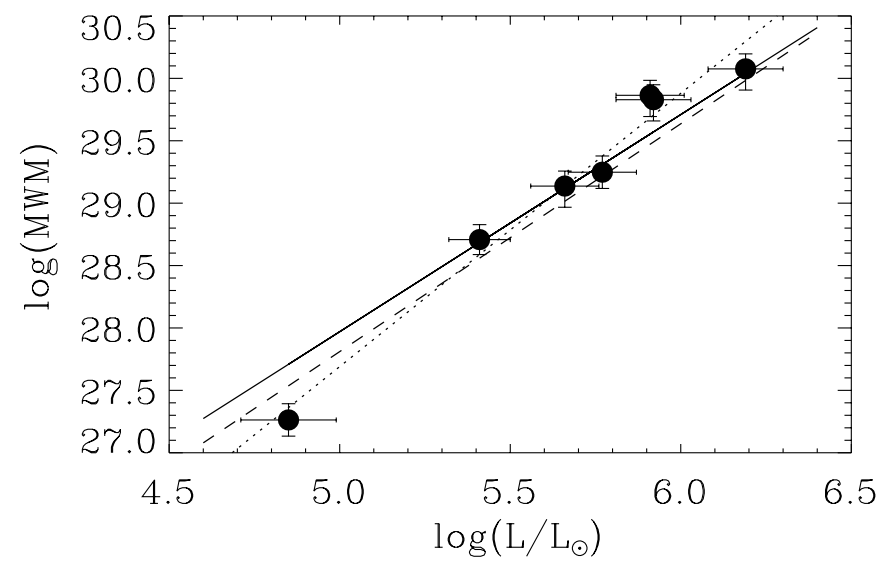

Fig. 15. The wind momentum-luminosity relationship obtained for the Cyg OB2 supergiants. The dotted line gives the regression obtained by including all stars, while the solid line gives the one obtained by including only Cyg OB2 \#8A, \#8C, \#4 and \#10. The dashed line very close to the solid one is the theoretical relation by Vink et al. (2000). The entry with the highest luminosity is Cyg OB2 \#8A and those close together are Cyg OB2 \#7 and \#11, the stars with extreme Of character.

even accounting for this uncertainty we cannot bring the position of these two stars into agreement with the WLR defined by the other four O stars.

However, taking into account the error bars, our data are still compatible with a unique relation including all seven stars. This is also shown in Fig. 15, where we display two different regressions, one including only Cyg OB2 \#8A, \#8C, \#4 and \#10, and one including all seven stars, respectively. Interestingly, the relation obtained by including only the $\mathrm{O}-$ supergiants, not shown for clarity, is still marginally compatible with the position of the B-supergiant.

In Fig. 16 we show a comparison of our data points with those obtained by Puls et al. (1996), updated by Herrero et al. (2000) for some entries. We see that both sets compare well, the most important difference being the fact that the scatter in our data points is lower. However, at present it is not possible to conclude whether this is a real improvement (as we have expected) or an artefact of the low number statistics. We note that the first possibility would imply that stars with extreme Of characteristics follow a slightly different relation than normal O stars, although we could not determine the reason for the relatively high position of Cyg OB2 \#7 and \#11.

In Table 4 we compare the coefficients we have obtained for the WLR (by weighted least-squares fits) using different samples (see above) as well as those quoted by Kudritzki \& Puls (2000) in their Table 2, and the coefficients of the theoretical relation provided by Vink et al. (2000). A correct error treatment would require to take into account errors in both axes and their correlation. This treatment is not simple and will be presented elsewhere (Markova \& Puls 2002). For the scope of the present paper we have adopted as error an average of the errors obtained when considering only those in the ordinate values (an underestimation) and when considering the errors in abscissa and ordinate as uncorrelated (an overestimation). Using both estimates for the errors, we have calculated the corresponding regression, where the resulting values for slope and

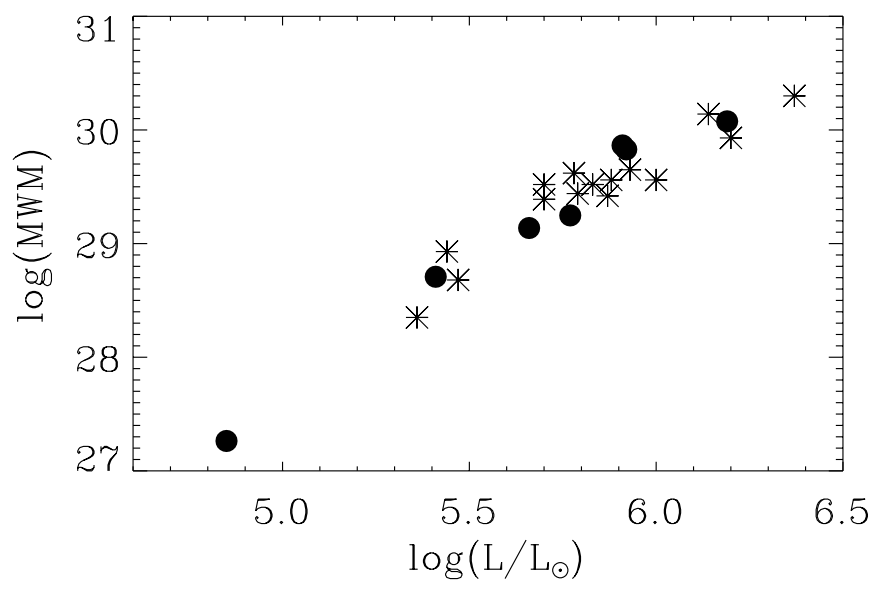

Fig. 16. A comparison of our results for Cyg OB2 OB-supergiants (filled dots) with the results by Puls et al. (1996) and Herrero et al. (2000).

offset turned out to be only marginally different. The final values quoted in Table 4 have been obtained from a straight average of these results.

We see that in spite of the visual agreement found in Fig. 16, our results differ significantly from those given by Kudritzki \& Puls (2000), in particular concerning the slope of the relation. Kudritzki \& Puls (2000) found a slope of $1.51 \pm 0.18$, while, when excluding the B supergiant, we obtain values from $1.74 \pm 0.24$ to $1.92 \pm 0.22$. In addition, our values for the vertical offset vary between 19.27 and 18.30, below the value of 20.69 of Kudritzki \& Puls (2000). Although the error bars allow for marginal agreement, the conclusion is that our relation is steeper and thus $\alpha_{\mathrm{eff}}^{\prime}(=1 / x)$ is smaller in our case. This indicates a different slope of the line-strength distribution function (see Puls et al. 2000 and Kudritzki \& Puls 2000, Sect. 4.1), i.e., a larger number of weak lines.

In contrast, we obtain very good agreement with the theoretical relation by Vink et al. (2000). Their relation, both in slope and offset, lies right between our relation when considering all supergiants or only the moderate O, Of stars, respectively. Our observations are thus compatible with a unique WLR for all O supergiants, but favour a separation of the extreme O, Of stars.

\section{Conclusions}

We have analyzed a homogeneous set of spectra of Cyg OB2 supergiants with spectral types ranging from $\mathrm{O} 3$ to $\mathrm{B} 1$. The analysis has been performed using a new version of the code presented by Santolaya-Rey et al. (1997) that includes an approximate treatement of metal line-blocking/blanketing. A test applied to the $09 \mathrm{~V}$ star $10 \mathrm{Lac}$ resulted in an excellent fit, at temperatures lower than those obtained previously (Herrero et al. 1992) and in agreement with other findings (Hubeny et al. 1998; Martins et al. 2002).

The fits to the supergiants are also satisfactory, except for the discrepancy between the fits of $\mathrm{H}_{\alpha}$ and other higher Balmer lines for stars with dense winds (see discussion in Sect. 6.1 of Herrero et al. 2000) and the pure absorption profile predicted 
Table 4. Coefficients for the wind momentum-luminosity relationship (see equation in p. 950) obtained in this work and taken from Kudritzki \& Puls (2000) and Vink et al. (2000).

\begin{tabular}{cccl}
\hline \hline $\log D_{0}$ & $x$ & $\alpha_{\text {eff }}^{\prime}$ & Comments \\
\hline $16.81 \pm 1.16$ & $2.18 \pm 0.21$ & $0.46 \pm 0.04$ & Using all Cyg OB2 stars \\
$18.30 \pm 1.82$ & $1.92 \pm 0.31$ & $0.52_{-0.07}^{+0.10}$ & Cyg OB2 \#2 not included \\
$19.27 \pm 1.82$ & $1.74 \pm 0.32$ & $0.58_{-0.09}^{+0.12}$ & Including only Cyg OB2 \#8A, \#8C, \#4, \#10 \\
$20.69 \pm 1.04$ & $1.51 \pm 0.18$ & $0.66 \pm 0.06$ & From Kudritzki \& Puls 2000 (Table 2) \\
$18.68 \pm 0.26$ & $1.826 \pm 0.044$ & $0.548 \pm 0.013$ & From Vink et al. 2000 (Eq. (15)) \\
\hline
\end{tabular}

for He II $\lambda 4686$ in Cyg OB2 \#8C instead of the P-Cygni profile observed.

We obtain temperatures that are cooler than in our previous analyses, as a consequence of the effects of sphericity, mass-loss and metal-line blocking/blanketing. Thanks to our coverage in spectral type, we can construct a temperature scale for $\mathrm{O}$-supergiants. This temperature scale is 4000 to $8000 \mathrm{~K}$ cooler at early types than the one presented by Vacca et al. (1996), mainly based on pure $\mathrm{H}-\mathrm{He}$ analyses. An important additional result is that at the same spectral subtype, Cyg OB2 \#8C and \#11 have a difference of $3000 \mathrm{~K}$ in their effective temperatures, reflecting their very different mass-loss rates. For the hotter stars, additional information based on a nitrogen temperature scale is needed, as the He lines become progressively either insensitive to changes in stellar parameters or simply too weak.

Models including metal line opacities result in cooler temperatures and similar radii than those using pure $\mathrm{H} / \mathrm{He}$ opacities, therefore giving smaller luminosities. Together with the blocking produced in the $(E) \mathrm{UV}$, the corresponding ionizing luminosities may change drastically, which will have a large impact on studies of the surrounding regions. However, each particular case has to be treated individually.

The results obtained indicate that Cyg OB2 stars are very massive. Only for two of the seven stars we found spectroscopic or evolutionary masses below $40 M_{\odot}$. Although there are still significant differences between spectroscopic and evolutionary masses, we do not find any obvious systematic pattern. Thus, at present we cannot conclude that there is a discrepancy between both sets of masses in Cyg OB2. However, this association is a very young one and the stars analyzed show only moderate projected rotational velocities: it might be possible that rotationally induced effects simply have had no sufficient time to become apparent. It is remarkable that we found an enhanced He abundance for only one of the stars, Cyg OB2 \#7.

Finally, we derived a new calibration of the wind momentum-luminosity relationship for $\mathrm{O}$ supergiants, including errors resulting from our analysis. Our data indicate that there might be a different relation for extreme Of and for moderate $\mathrm{O}$, Of stars, respectively, perhaps indicating different ionization conditions or clumping in the wind. Considering only the moderate $\mathrm{O}$, Of stars, we obtain a very low scatter in the relation, but this might reflect only the low number statistics. In fact, our still limited sample is also consistent with a unique relation including all stars, even the B supergiant. Clearly, more data are needed to disentangle whether the large modified wind momentum rate we derive for the extreme Of stars is a real, physical effect or just the result of our poor statistics.

Acknowledgements. We thank an annonymous referee for very valuable suggestions that helped to improve the paper. FNP thanks the spanish MCyT for grants ESP98-1351 and PNAYA2000-1784 within the Ramón y Cajal Program. AHD thanks support from the spanish MCyT under project PNAYA2001-0436 that includes EU FEDER funds.

\section{References}

Abbott, D. C., \& Lucy, L. B. 1985, ApJ, 288, 679

Bianchi, L., \& García, M. 2002, ApJ, in press

Bieging, J. H., Abbott, D. C., \& Churchwell, E. B. 1989, ApJ, 340, 518

Bresolin, F., Kudritzki, R. P., Lennon, D. J., et al. 2002, ApJ, in press Bohlin, R. C. 1975, ApJ, 200, 402

Conti, P. S., \& Alschuler, W. R. 1971, ApJ, 170, 325

Crowther, P. A., \& Bohannan, B. 1997, A\&A, 317, 532

Crowther, P. A., Hillier, D. J., Evans, C. J., et al. 2002, ApJ, in press

Friend, D. B., \& Abbott, D. C. 1986, ApJ, 311, 701

Fullerton, A. W., Crowther, P. A., De Marco, O., et al. 2000, ApJ, 538, L43

Groenewegen, M. A. T., \& Lamers, H. J. G. L. M. 1989, A\&AS, 79, 359

Hamann, W. R. 1981, A\&A, 93, 353

Haser, S. M. 1995, Ph.D. Thesis, Universitäts-Sternwarte der Ludwig-Maximillian Universität, München

Herrero, A., Kudritzki, R. P., Vílchez, J. M., et al. 1992, A\&A, 261, 209

Herrero, A., Corral, L. J., Villamariz, M. R., \& Martín, E. L. 1999, A\&A, 348, 542

Herrero, A., Puls, J., Corral, L. J., et al. 2001, A\&A, 366, 623

Herrero, A., Puls, J., \& Villamariz, M. R. 2000, A\&A, 354, 193

Hillier, D. J., \& Miller, D. L. 1998, ApJ, 496, 407

Howarth, I. D., \& Prinja, R. K. 1989, ApjS, 69, 527

Hubeny, I., Heap, S. R., \& Lanz, T. 1998, ASP Conf. Ser., 131, 108

Hubeny, I., \& Lanz, T. 1995, ApJ, 439, 875

Jenkins, E. B. 1970, in on Ultraviolet Stellar Spectra and Ground Based Observations, ed. L. Houziaux, \& H. E. Butler (Dordrecht: Reidel), IAU Symp., 36, 281

Knödlseder, J. 2000, A\&A, 360, 539

Kudritzki, R. P., \& Puls, J. 2000, ARA\&A, 38, 613

Kudritzki, R. P., Puls, J., Lennon, D. J., et al. 1999, A\&A, 350, 970

Kudritzki, R. P., Pauldrach, A. W. A., Puls, J., \& Abbott, D. C. 1989, A\&A, 219, 205

Kurucz, R. L. 1992, in The Stellar Populations of Galaxies, ed. B. Barbuy, \& A. Renzini (Kluwer, Dordrecht), IAU Symp., 149,225 
Lamers, H. J. G. L. M., Haser, S., de Koter, A., \& Leitherer, C. 1999, ApJ, 516, 872

Leitherer, C., Hefele, H., Stahl, O., \& Wolf, B. 1982, A\&A, 108, 102 Markova, N., \& Puls, J. 2002, in prep.

Martins, F., Schaerer, D., \& Hillier, D. J. 2002, A\&A, 382, 999

Massey, P., \& Thompson, A. B. 1991, AJ, 101, 1408

Mathys, G. 1988, A\&AS, 76, 427

McCarthy, J. K., Kudritzki, R. P., Lennon, D. J., et al. 1997, ApJ, 482, 757

Meynet, G., \& Maeder, A. 2000, A\&A, 361, 101

Najarro, F. 2002, in prep.

Pauldrach, A. W. A., Lennon, M., Hoffmann, T. L., et al. 1998, PASPC, 131, 258

Pauldrach, A. W. A., Hoffmann, T. L., \& Lennon, M. 2001, A\&A, 375, 161

Puls, J. 1991, A\&A, 248, 581

Puls, J. 2002, in prep.

Puls, J., Owocki, S. P., \& Fullerton, A. W. 1993, A\&A, 279, 457

Puls, J., Kudritzki, R. P., Herrero, A., et al. 1996, A\&A, 305, 171

Puls, J., Springmann, U., \& Lennon, M. 2000, A\&AS, 141, 23

Santolaya-Rey, A. E., Puls, J., \& Herrero, A. 1997, A\&A, 323, 488

Schaerer, D., \& Schmutz, W. 1994, A\&A, 288, 231

Schaller, G., Schaerer, D., Meynet, G., \& Maeder, A. 1992, A\&AS, 96, 269
Schmutz, W. 1991, in Stellar Atmospheres: Beyond Classical Models, ed. L. Crivellari, I. Hubeny, \& D. G. Hummer, NATO ASI Ser. C, 341 (Kluwer, Dordrecht), 191

Schulte, D. H. 1958, ApJ, 128, 41

Shull, J. M., \& Van Steenberg, M. E. 1985, ApJ, 294, 599

Scuderi, S., Panagia, N., Stanghellini, C., Trigilio, C., \& Umana, G. 1998, A\&A, 332, 251

Taresch, G., Kudritzki, R. P., Hurwitz, M., et al. 1997, A\&A, 321, 531

Urbaneja, M. A., Herrero, A., Kudritzki, R. P., et al. 2002, A\&A, 386, 1019

Vacca, W. D., Garmany, C. D., \& Shull, J. M. 1996, ApJ, 460, 914

Villamariz, M. R., Herrero, A., Butler, K., \& Becker, S. R. 2002, A\&A, 388, 940

Vink, J. S., de Koter, A., \& Lamers, H. J. G. L. M. 2000, A\&A, 362, 295

Walborn, N. R. 1973, AJ, 78, 1067

Walborn, N. R., Howarth, I. D., Lennon, D. J., et al. 2002, AJ, 123, 2754

Walborn, N. R., Lennon, D. J., Heap, S. R., et al. 2000, PASP, 112, 1243

Walborn, N. R., Nichols-Bohlin, J., \& Panek, R. J. 1985, NASA Ref. Pub., 1155

Waldron, W. L., Corcoran, M. F., Drake, S. A., \& Smale, A. P. 1998, ApJS, 118, 217 